\title{
Necessary and Sufficient Conditions for the Noninvertibility of Fundamental Solution Matrices of a Discontinuous System*
}

\author{
Federico Bizzarri ${ }^{\dagger}$, Alessandro Colombo $\ddagger$, Fabio Dercole ${ }^{\ddagger}$, and Giancarlo Storti Gajani ${ }^{\ddagger}$
}

\begin{abstract}
In discontinuous systems, the fundamental solution matrix of the linearized dynamics about a reference trajectory can be noninvertible. This feature can be exploited, for instance, to design robust control algorithms, to synchronize a network, or to stabilize otherwise unstable or chaotic dynamics. In this paper we classify all the phenomena that cause rank defect in the fundamental solution matrix of a generic discontinuous system. We relate these phenomena to simple geometric conditions at a point of vector field switching, sliding, or impact, and we derive necessary and sufficient conditions for the rank defect. This constitutes a valuable tool to detect flow noninvertibility or to purposefully include it in the design of a system. In terms of Lyapunov exponents, the singularity of the fundamental solution matrix means that an infinitesimal sphere of initial perturbations is mapped onto a lower-dimensional ellipsoid. This consequently reduces the number of finite exponents and, most remarkably, makes them depend on the full history of the reference trajectory used for the computation. We introduce a numerical procedure which allows the computation of the Lyapunov exponents by resorting to a properly reduced variational system. The validity of the approach is verified in two examples.
\end{abstract}

Key words. hybrid system, piecewise smooth system, dimension reduction, noninvertibility, Lyapunov exponents, saltation matrix, discontinuity mapping

AMS subject classifications. 34D08, 34A36, 34A38, 37M25, 93D99

DOI. $10.1137 / 140959031$

1. Introduction. Discontinuous systems are commonly used in several fields of science and engineering, e.g., in mechanics, to model impacts and friction [12]; in analog-digital electronics [8], to model switches [7, 9, 16, 29]; in many control systems [38]; in ecology, to model optimal foraging behaviors $[24,25]$; in evolutionary biology, to describe evolutionary reversals induced by switching in the underlying ecological regime $[22,23]$; in neuroscience, to take neuron firing and reset into account [40]; in biological and medical systems [1]; and in the social sciences and finance, to mimic decision processes and behavioral strategies [19, 14].

In the presence of discontinuities the forward or backward evolution of a given initial condition may not be unique. Forward-time nonuniqueness, which is typically a consequence of an extreme sensitivity of the modelled phenomenon, poses numerous problems in terms of the analysis of a system, first and foremost regarding the definition of a so-

${ }^{*}$ Received by the editors February 28, 2014; accepted for publication (in revised form) by H. Dankowicz October 7, 2015; published electronically January 26, 2016.

http://www.siam.org/journals/siads/15-1/95903.html

${ }^{\dagger}$ Department of Electronics, Information, and Bioengineering, Politecnico di Milano, 20133 Milano, Italy, and Advanced Research Center on Electronic Systems for Information and Communication Technologies E. De Castro (ARCES), University of Bologna, 40125 Bologna, Italy (federico.bizzarri@polimi.it).

‡Department of Electronics, Information, and Bioengineering, Politecnico di Milano, 20133 Milano, Italy (alessandro.colombo@polimi.it, fabio.dercole@polimi.it, giancarlo.stortigajani@polimi.it). The work of the third author was supported by the Italian Ministry for University and Research (under contract FIRB RBFR08TIA4). 
lution. Noninvertibility - the infinitely fast convergence of multiple trajectories - is, on the other hand, a relatively innocuous and sometimes useful feature, used, for example, as a means to simplify the analysis through dimension reduction [13], to design robust control laws such as in sliding-mode control [51], or to ensure the synchronization of a network [45] or the regularization of otherwise chaotic dynamics [42].

Restricting to the cases where forward-time uniqueness is ensured, in this paper we discuss the necessary and sufficient conditions for noninvertibility of the linearized dynamics of a discontinuous system about a reference trajectory. The result is a set of simple rules, characterized both algebraically and geometrically, to detect noninvertibility, providing a useful tool for the designer who wishes to exploit it. Since the invertibility of a nonlinear system is a local property of a trajectory, a local, variational analysis is best suited for the task at hand. We thus develop our discussion around the concept of a fundamental solution matrix, that is, the first-order expansion of the dynamics of perturbations around the reference trajectory. As a by-product of these results, the discussion is naturally extended to Lyapunov exponents (LEs) as a means to measure the average exponential rates of expansion or contraction of infinitesimal perturbations inside and transverse to the system's attractors. Unlike in smooth systems, where the LEs computed along trajectories converging to the same attractor (almost everywhere) coincide with the attractor's exponents, in discontinuous systems the history of the discontinuities crossed by a trajectory may affect the resulting LEs, which are therefore a property of the whole trajectory and not just of its limit set.

The computation of the fundamental solution matrix and of the LEs for model-explicit, nonlinear, and finite-dimensional dynamical systems is based on numerical procedures that require the system to be ruled by a sufficiently smooth vector field [31]. In particular, the computation is based on the integration of a variational system, describing the linearized evolution of a perturbation around the reference trajectory, and requires the system's Jacobian to be continuous. When a model is discontinuous, for example, to represent a sudden change in the dynamics or a fast process such as a mechanical impact, or because nonlinear laws have been approximated by piecewise linear ones, the standard approaches cannot be applied.

Extensions of the basic algorithm for the computation of the fundamental solution matrix and of the LEs to several classes of discontinuous systems have been proposed, starting with Müller [46] in 1995. Müller considered generic $n$-dimensional vector fields switching when the system state transversely intersects $(n-1)$-dimensional smooth manifolds in state space, by introducing linear corrections in the linearized equations at the times of switching of the reference trajectory. This approach was applied, for instance, by Hinrichs, Oestreich, and Popp [37] in 1997. Müller did not consider the possibility of sliding along the switching manifolds or of impacts, i.e., jumps in state space induced by impulsive behavior of the system equations.

Müller's corrections belong to the more general framework of discontinuity mappings, introduced by Aizerman and Gantmakher in 1958 [2] and Filippov in 1960 [32] (see also the 1988 book by Filippov [33]) and generalized to full nonlinearity by Nordmark in 1991 [47]. A discontinuity mapping provides the correction that must be applied to all perturbations of a reference trajectory, at a point of discontinuity, ${ }^{1}$ in order to integrate them as if they were

\footnotetext{
${ }^{1}$ In what follows we will refer to "time of discontinuity" and "point of discontinuity" of a given trajectory or to "discontinuity manifold" without distinction between switching and impacting phenomena. The discontinuity may occur in the vector field (switching) as well as in the state of the system (impact).
} 
reaching the discontinuity all at the same time. The Jacobian of the discontinuity mapping at the point of discontinuity of the reference trajectory is the correction factor resetting the state of the variational system at the time of discontinuity. This Jacobian is known in the literature of piecewise smooth systems as the saltation matrix [28]. Analogous corrections were implemented by de Souza and Caldas [21] for mechanical systems with impacts and, more recently, by Zhou et al. [54] for a particular type of resetting impact typical in neuroscience. Finally, Dieci and Lopez [30] discussed the variational system for piecewise-smooth discontinuous systems (so-called Filippov systems), also in the presence of sliding. The saltation matrix at the onset of sliding is singular, and this makes the fundamental solution matrix singular as well. The singularity of the fundamental solution matrix means that an infinitesimal $n$-dimensional sphere of initial perturbations is mapped onto a lower-dimensional ellipsoid; viz., some perturbation dimensions are lost. Dieci and Lopez showed that multiple sliding segments on $(n-1)$-dimensional manifolds generically induce a loss of one dimension only, so that one LE is expected to diverge to $-\infty$, whereas in general $m$ dimensions are lost when sliding on an $(n-m)$-dimensional manifold.

In order to discuss the mathematical intricacies of the fundamental solution matrix and LEs of discontinuous systems in the greatest possible generality, we have set our paper in the framework of hybrid systems. These are continuous time evolution processes interfaced with discrete time decision processes [11]. They can easily be used to describe most of the discontinuous models mentioned above and have been shown to be equivalent to a number of other relevant discontinuous system formalisms [36].

Specifically, we classify all the phenomena that can cause rank defect of a saltation matrix in a generic hybrid system. This includes systems that may exhibit vector field switching, sliding, impacts, tangencies of the vector fields with the discontinuity manifold (with some restrictions, as we see in the next paragraph), and singular reset maps at impact. For each scenario we provide means to determine the rank defect that is generically expected. We adapt the most popular algorithm for LE computation (discrete QR [31]) to handle singular fundamental solution matrices.

We do not discuss the theoretical problems related to the definition of solutions of the hybrid system [28], and we assume solutions to be uniquely defined forward in time. This clearly excludes some relevant cases, for instance, those where a discontinuity causes infinite expansion (escaping) and generates multiple solutions for a given initial condition [41, 18, 17]. Analogously, we do not consider the cases where the reference trajectory reaches the discontinuity manifold tangentially [27] (though we allow it to leave the manifold tangentially), those where it reaches simultaneously two or more manifolds [39, 26, 50, 15], and those in which it reaches a manifold infinite times in a finite amount of time [53]. In these cases, the discontinuity mapping may not be differentiable, and the saltation matrix may be undefined. As a consequence, the usual definitions of the fundamental solution matrix and LEs cannot be used. Suitable indicators for the expansion or contraction of infinitesimal perturbations should then be defined based on a nonlinear approximation of the hybrid dynamics. It is worth noting that the evolution of a single perturbation in the case of simultaneous multiple contacts of the reference trajectory has been considered by Dieci and Lopez [30] and later extended [10] to $n$ canonical perturbations to obtain a heuristic computation of Lyapunov indicators. 
The paper is organized as follows. In section 2 the concept of the fundamental solution matrix for a hybrid system and the role played by the saltation matrices at the discontinuities are recalled. Section 3 contains the main theoretical results and classifies the cases leading to a singular saltation matrix, while section 4 particularizes the results for Filippov systems. Section 5 presents our (discrete QR) procedure for LEs computation in detail. The proposed algorithm is applied in section 6 in two case studies. Conclusions and further directions of research are discussed in section 7 .

In the following, we use boldface letters for column vectors, the superscript " $T$ " for transposition, and a subscript " $\mathrm{x}$ " for differentiation with respect to $\mathbf{x} . H_{\mathbf{x}}(\mathbf{x}), H: X \subseteq \mathbb{R}^{n} \rightarrow \mathbb{R}$, is a row vector. $I$ is the identity matrix, whereas $\operatorname{ker} A$ and $\operatorname{im} A$ denote the null space and the image of matrix $A$.

2. The fundamental solution matrix in a hybrid system. Let us consider a dynamical system whose dynamics is described by

$$
\dot{\mathbf{x}}(t)=f(\mathbf{x}(t)),
$$

where $\mathbf{x}(t) \in X \subseteq \mathbb{R}^{n}$ and $f: X \rightarrow \mathbb{R}^{n}$ is a smooth vector field. Given the initial condition $\mathbf{x}_{0}$ at time $t_{0}$, we call $\varphi\left(\mathbf{x}_{0}, t, t_{0}\right): \mathbb{R}^{n} \times \mathbb{R} \times \mathbb{R} \rightarrow \mathbb{R}^{n}$ the flow of $\mathbf{x}_{0}$ at time $t$, i.e., $\mathbf{x}(t)=$ $\varphi\left(\mathbf{x}_{0}, t, t_{0}\right)$. Given a reference initial condition $\mathbf{x}_{0}:=\mathbf{x}\left(t_{0}\right)$ and its forward-time trajectory $\mathbf{x}(t)$, the fundamental solution matrix $\psi\left(t, t_{0}\right)$ is defined as the unique matrix that satisfies the relation

$$
\mathbf{x}^{\prime}(t)-\mathbf{x}(t)=\psi\left(t, t_{0}\right)\left(\mathbf{x}^{\prime}\left(t_{0}\right)-\mathbf{x}_{0}\right)+O\left(\left\|\mathbf{x}^{\prime}\left(t_{0}\right)-\mathbf{x}_{0}\right\|^{2}\right)
$$

for any possible infinitesimal perturbation $\mathbf{x}^{\prime}\left(t_{0}\right)$ of $\mathbf{x}_{0}$. The fundamental solution matrix is therefore associated with the reference trajectory originating at $\mathbf{x}\left(t_{0}\right)$. For the smooth system (2.1), it is obtained as the solution of the matrix initial value problem

$$
\dot{\psi}\left(t, t_{0}\right)=J(\mathbf{x}(t)) \psi\left(t, t_{0}\right), \quad \psi\left(t_{0}, t_{0}\right)=I,
$$

where $J(\mathbf{x}(t))$ is the Jacobian of $f$ at $\mathbf{x}(t)$.

In order to define the fundamental solution matrix of a hybrid system's trajectory, we formally define hybrid systems as follows.

Definition 2.1. A hybrid system is a tuple $\{X, Q, F, G, R\}$, where $X \subseteq \mathbb{R}^{n}$ is the space of the continuous variables $\mathbf{x} ; Q \subseteq \mathbb{N}$ is a set of values for the discrete variable $q ; F: X \times Q \rightarrow \mathbb{R}^{n}$ is a collection of smooth vector fields $F_{q} ; G \subset X \times Q$ is a collection of discontinuity sets (or guards), each composed of possibly intersecting $(n-1)$-dimensional smooth manifolds in $X$; and $R: G \rightarrow X \times Q$ is a collection of reset maps.

The vector fields describe the continuous dynamics of the state, while the reset maps trigger discontinuous transitions in the state and in the vector field itself, whenever the state hits a discontinuity set. Let us denote by $G_{q} \subset X$ and $R_{q}: G_{q} \rightarrow X$, respectively, the collection of discontinuity manifolds and the $X$-component of the reset map corresponding to a specific value of the discrete variable $q$. The map $R_{q}$ needs to be defined only at points $\mathrm{x}$ that belong to $G_{q}$. However, many of the results that follow use the differential $R_{q, \mathbf{x}}(\mathbf{x})$, and in order to compute this differential we must consider a smooth extension of $R_{q}$ to an 
open neighborhood of $\mathrm{x} \in G_{q}$. As we will see in Corollary 3.6, all our results are completely independent of the choice of extension, so we can assume without loss of generality that such an extension is given. Let us also partition $G_{q}$ into the subset $\Xi_{q}$ of points belonging to only one smooth discontinuity manifold and the subset $\Upsilon_{q}$ of points belonging to the intersection of two or more manifolds.

Definition 2.2. A trajectory of a hybrid system with initial conditions $\left(\mathbf{x}_{0}, q\right) \in X \times Q$ is an integral $\mathbf{x}(t)$ of the vector field $F_{q}$ up to the time $\bar{t}$, where $\overline{\mathbf{x}}:=\mathbf{x}(\bar{t}) \in G_{q}$. Upon hitting the discontinuity set, the state $(\mathbf{x}, q)$ is reset according to the reset map $\left(R_{q}(\overline{\mathbf{x}}), q^{\prime}\right)=R(\overline{\mathbf{x}}, q)$, and the integration proceeds following the new vector field $F_{q^{\prime}}$.

To obtain a sensible definition of the fundamental solution matrix we need to assume some restrictions on the geometry of the reference trajectory at the points of discontinuity. These restrictions are collected in assumptions (H.0), (H.1), and (H.2) which follow. First, as anticipated in the introduction, we assume uniqueness of the (hybrid)-flow in forward time:

(H.0) The hybrid-flow $\varphi\left(\mathbf{x}^{\prime}\left(t_{0}\right), t, t_{0}\right)$ for $\mathbf{x}^{\prime}\left(t_{0}\right)$ in a neighborhood of the reference initial condition $\mathbf{x}_{0}$ is a single-valued function of $t$ for all $t \geq t_{0}$.

Second, when an infinite number of resets occur in a finite time, Definition 2.2 cannot be applied. Extensions of Definitions 2.1 and 2.2 that can overcome this obstacle are studied in the hybrid systems literature [49] but fall outside the scope of this paper. In what follows, we limit our analysis to

(H.1) trajectories undergoing a finite number of resets in any finite time interval.

Third, we assume a transversality condition on the points of intersection of the reference trajectory with the discontinuity:

(H.2) Trajectory $\mathbf{x}(t)$ never reaches $\Upsilon_{q} \subset G_{q}$, and it reaches $\Xi_{q} \subset G_{q}$ transversely.

Indeed, at a point of discontinuity the matrix function $J(\mathbf{x}(t))$ is discontinuous, and a straightforward solution of (2.3) in the sense of Carathéodory (i.e., a continuous function $\psi\left(t, t_{0}\right)$ whose time derivative satisfies (2.3) almost everywhere) does not satisfy (2.2): the vector field switching and/or the state impact causes a sudden jump in the entries of the matrix $\psi$. However, when the hybrid-flow $\varphi\left(\mathbf{x}_{0}, t, t_{0}\right)$ is differentiable in $\mathbf{x}_{0}$ for almost every $t$, letting

$$
\begin{aligned}
& \psi^{-}\left(\bar{t}, t_{0}\right):=\lim _{t \rightarrow \bar{t}^{-}} \psi\left(t, t_{0}\right), \\
& \psi^{+}\left(\bar{t}, t_{0}\right):=\lim _{t \rightarrow \bar{t}^{+}} \psi\left(t, t_{0}\right),
\end{aligned}
$$

the matrix $\psi^{+}\left(\bar{t}, t_{0}\right)$ can be expressed as a linear map of $\psi^{-}\left(\bar{t}, t_{0}\right)$, which allows the writing of $(2.3)$ as an equation piecewise-smooth in $t$. The hybrid-flow may not be differentiable in $\mathbf{x}_{0}$ for $t>\bar{t}$ if the trajectory $\mathbf{x}(t)$ is tangent to the discontinuity manifold at $\overline{\mathbf{x}}$, or if it simultaneously reaches two or more manifolds at $\bar{t}$ (i.e., if $\overline{\mathbf{x}} \in \Upsilon_{q}$ ). ${ }^{2}$ In these two cases we lose the traditional concept of fundamental solution matrix [30]. In contrast, if these two cases are excluded, that is, provided that (H.2) holds, Aizerman and Gantmakher [2] observed that the differentiability of the flow in $\mathbf{x}_{0}$ is ensured.

In general, it is not easy to prove that assumptions (H.0)-(H.2) hold globally for a given system. However, all assumptions can be easily checked to hold in a neighborhood of a

\footnotetext{
${ }^{2}$ Seminal results concerning discontinuous systems, in which the trajectories intersect several surfaces of discontinuity at the same time, can be found in [39].
} 
specific trajectory, numerically or analytically computed. We assume throughout the paper that (H.0)-(H.2) hold in a neighborhood of the reference trajectory.

Representing the discontinuity set as the zero set of a smooth function $H: X \rightarrow \mathbb{R}$ with $H_{\mathbf{x}}(\overline{\mathbf{x}}) \neq \mathbf{0},{ }^{3}$ the saltation matrix is given by

$$
\mathcal{S}:=R_{q, \mathbf{x}}(\overline{\mathbf{x}})+\frac{F_{q^{\prime}}\left(R_{q}(\overline{\mathbf{x}})\right)-R_{q, \mathbf{x}}(\overline{\mathbf{x}}) F_{q}(\overline{\mathbf{x}})}{H_{\mathbf{x}}(\overline{\mathbf{x}}) F_{q}(\overline{\mathbf{x}})} H_{\mathbf{x}}(\overline{\mathbf{x}})
$$

(a proof of (2.4) can be found in [28]; note that we have $H_{\mathbf{x}}(\overline{\mathbf{x}}) F_{q}(\overline{\mathbf{x}}) \neq 0$ by the transversality assumption (H.2)). It is then possible to write

$$
\psi^{+}\left(\bar{t}, t_{0}\right)=\mathcal{S} \psi^{-}\left(\bar{t}, t_{0}\right) .
$$

Unlike in smooth systems, where the group structure of the flow ensures invertibility of the fundamental solution matrix, in a hybrid system formalized as in Definition 2.1, the hybridflow under (H.0)-(H.2) forms a semigroup (solutions are not necessarily unique backward in time), and the fundamental solution matrix need not be invertible, due to the singularity of one or more of the saltation matrices along the trajectory $\mathbf{x}(t)$. The rank defect of the elements at the left- and right-hand sides of (2.5) obey the following general rule (which we report without proof).

Proposition 2.3. Given two matrices $\mathrm{A}, \mathrm{B} \in \mathbb{R}^{n \times n}$ and such that $\operatorname{dim} \operatorname{ker} \mathrm{A}=s>0$ and $\operatorname{dim} \operatorname{ker} \mathrm{B}=r \geq 0, \operatorname{dim} \operatorname{ker} \mathrm{AB}=r+m$, where $m:=\operatorname{dim}(\operatorname{ker} \mathrm{A} \cap \operatorname{im} \mathrm{B}) \leq s$.

Therefore, when at time $\bar{t}$ the reference trajectory crosses a discontinuity with a saltation matrix $\mathcal{S}$ with rank defect $s$, the rank defects of $\psi^{-}\left(\bar{t}, t_{0}\right)$ and $\psi^{+}\left(\bar{t}, t_{0}\right)$ are $r \geq 0$ and $r+m \leq$ $r+s$, respectively. When several singular saltation matrices are encountered, what typically matters is the saltation matrix with largest rank defect. This follows from the following proposition.

Proposition 2.4. Assuming

$$
\operatorname{dim}\left(\operatorname{ker} \mathcal{S}_{k} \cap \operatorname{im} \psi^{-}\left(\bar{t}_{k}, t_{0}\right)\right)=\max \left\{0, \operatorname{dim}\left(\operatorname{ker} \mathcal{S}_{k}\right)+\operatorname{dim}\left(\operatorname{im} \psi^{-}\left(\bar{t}_{k}, t_{0}\right)\right)-n\right\},
$$

where $\mathcal{S}_{k}, k=1,2, \ldots$, are the saltation matrices encountered along the reference trajectory from $\mathbf{x}_{0}$ to $\mathbf{x}(t)$ at times $\bar{t}_{k}$, we have that

$$
\operatorname{dim} \operatorname{ker} \psi\left(t, t_{0}\right)=\max _{k} \operatorname{dim} \operatorname{ker} \mathcal{S}_{k} .
$$

Proof. For each $k$, let

$$
s_{k}:=\operatorname{dim} \operatorname{ker} \mathcal{S}_{k} \quad \text { and } \quad r_{k}:=\operatorname{dim} \operatorname{ker} \psi^{-}\left(\bar{t}_{k}, t_{0}\right) .
$$

Then, by the proposition's assumption,

$$
\begin{aligned}
m_{k} & :=\operatorname{dim}\left(\operatorname{ker} \mathcal{S}_{k} \cap \operatorname{im} \psi^{-}\left(\bar{t}_{k}, t_{0}\right)\right) \\
& =\max \left\{0, s_{k}+\left(n-r_{k}\right)-n=s_{k}-r_{k}\right\},
\end{aligned}
$$

\footnotetext{
${ }^{3}$ Note that the discontinuity set $G_{q}$ is a collection of possibly intersecting $(n-1)$-dimensional smooth manifolds. However, by hypothesis (H.2), for every $\mathbf{x} \in \Xi_{q}$, the manifold is smooth in a sufficiently small neighborhood of $\mathbf{x}$.
} 
so that by Proposition 2.3 and since $r_{1}=0$ we have $\operatorname{dim} \operatorname{ker} \psi^{+}\left(\bar{t}_{k}, t_{0}\right)=\max _{i \in\{1, \ldots, k\}} s_{i}$.

Note that the assumption in Proposition 2.4 is the standard transversality condition for two subspaces in an ambient space of dimension $n$, and it is, as such, typically satisfied. However, higher rank defects can occur in special cases, when $\operatorname{ker} \mathcal{S}_{k}$ and $\operatorname{im} \psi^{-}\left(\bar{t}_{k}, t_{0}\right)$ are in nongeneric (i.e., not transverse) position. One such case is described at the end of section 4.

3. Singularity of the saltation matrix. In this section, we provide necessary and sufficient conditions for the singularity of the saltation matrix $\mathcal{S}$ in (2.4), we discuss their geometric meaning, and we characterize, case by case, the corresponding null space of $\mathcal{S}$.

Consider a trajectory $\mathbf{x}(t)$ reaching $\Xi_{q}$ transversely at point $\overline{\mathbf{x}}$ (recall hypothesis (H.2)). The discontinuity set $\Xi_{q}$ locally forms an $(n-1)$-dimensional smooth manifold, which we denote $\mathcal{H}$, described by the zero set of the function $H$. Let $\mathcal{H}^{\prime}$ be the image of $\mathcal{H}$ through the reset map $R_{q}$. Note that the tangent space to $\mathcal{H}^{\prime}$ at $R_{q}(\overline{\mathbf{x}})$ is the image through $R_{q, \mathbf{x}}(\overline{\mathbf{x}})$ of the tangent space to $\mathcal{H}$ at $\overline{\mathbf{x}}$. Thus, vectors tangent to $\mathcal{H}^{\prime}$ at $R_{q}(\overline{\mathbf{x}})$ necessarily belong to $\operatorname{im} R_{q, \mathbf{x}}(\overline{\mathbf{x}})$. When $R_{q, \mathbf{x}}(\overline{\mathbf{x}})$ is singular, we use $p:=\operatorname{dim} \operatorname{ker} R_{q, \mathbf{x}}(\overline{\mathbf{x}})$ and $p_{\mathcal{H}}:=\operatorname{dim}\left(\operatorname{ker} R_{q, \mathbf{x}}(\overline{\mathbf{x}}) \cap \operatorname{ker} H_{\mathbf{x}}(\overline{\mathbf{x}})\right)$, so that $\operatorname{dim} \mathcal{H}^{\prime}=\operatorname{dim} \mathcal{H}-p_{\mathcal{H}}$ is equal to $n-p$ if $\operatorname{ker} R_{q, \mathbf{x}}(\overline{\mathbf{x}}) \not \subset \operatorname{ker} H_{\mathbf{x}}(\overline{\mathbf{x}})$ (because necessarily $p_{\mathcal{H}}=p-1$ ), and is $n-1-p$ otherwise (because obviously $p_{\mathcal{H}}=p$ ).

We have the following result. ${ }^{4}$

Theorem 3.1. The matrix $\mathcal{S}$ in (2.4) is singular if and only if at least one of the following conditions is satisfied:

(i) $F_{q^{\prime}}\left(R_{q}(\overline{\mathbf{x}})\right)$ is tangent to $\mathcal{H}^{\prime}$ at $R_{q}(\overline{\mathbf{x}})$;

(ii) $\operatorname{dim} \mathcal{H}^{\prime}<\operatorname{dim} \mathcal{H}$.

Note that the case in which $R_{q}(\overline{\mathbf{x}})$ is an equilibrium of the vector field $F_{q^{\prime}}$ (i.e., $F_{q^{\prime}}\left(R_{q}(\overline{\mathbf{x}})\right)=$ $\mathbf{0})$ is included in case (i), while the case $F_{q}(\overline{\mathbf{x}})=0$ is excluded by (H.2). To prove the above theorem, we reformulate conditions (i) and (ii) algebraically in Lemmas 3.3 and 3.4, and we use the following result (Proposition 3.2.1 in [34]).

Proposition 3.2. If $\mathbf{b} \in \operatorname{im} A$, then there exists a unique vector $\mathbf{w}$ such that

$$
\mathbf{w} \in \operatorname{im} A^{T}, \quad A \mathbf{w}=\mathbf{b} .
$$

It can be found as the solution of the nonsingular system

$$
\left[\begin{array}{cc}
A & Z^{\prime} \\
Z^{T} & 0
\end{array}\right]\left[\begin{array}{l}
\mathbf{w} \\
\mathbf{r}
\end{array}\right]=\left[\begin{array}{l}
\mathbf{b} \\
\mathbf{0}
\end{array}\right]
$$

where the columns of the matrices $Z$ and $Z^{\prime}$ form a base of $\operatorname{ker} A$ and $\operatorname{ker} A^{T}$, respectively.

Note that in the above proposition $\mathbf{r}=\mathbf{0}$ always, and that we can equivalently write $\mathbf{w}=A^{\dagger} \mathbf{b}$, where $A^{\dagger}$ is the Moore-Penrose pseudoinverse of $A$ [4].

Lemma 3.3. Case (i) in Theorem 3.1 is equivalent to the following mutually exclusive sets of conditions:

(i.a) (i.a.1) $\operatorname{dim} \operatorname{ker} R_{q, \mathbf{x}}(\overline{\mathbf{x}})=0$,

(i.a.2) $H_{\mathbf{x}}(\overline{\mathbf{x}}) R_{q, \mathbf{x}}(\overline{\mathbf{x}})^{-1} F_{q^{\prime}}\left(R_{q}(\overline{\mathbf{x}})\right)=0$;

(i.b) (i.b.1) $\operatorname{ker} R_{q, \mathbf{x}}(\overline{\mathbf{x}}) \not \subset \operatorname{ker} H_{\mathbf{x}}(\overline{\mathbf{x}})$,

(i.b.2) $F_{q^{\prime}}\left(R_{q}(\overline{\mathbf{x}})\right) \in \operatorname{im} R_{q, \mathbf{x}}(\overline{\mathbf{x}})$;

\footnotetext{
${ }^{4}$ Hypotheses (H.0)-(H.2) are assumed to hold in a neighborhood of the reference trajectory $\mathbf{x}(t)$.
} 
(i.c) (i.c.1) $\operatorname{dim} \operatorname{ker} R_{q, \mathbf{x}}(\overline{\mathbf{x}})>0$,

(i.c.2) $\operatorname{ker} R_{q, \mathbf{x}}(\overline{\mathbf{x}}) \subset \operatorname{ker} H_{\mathbf{x}}(\overline{\mathbf{x}})$,

(i.c.3) $F_{q^{\prime}}\left(R_{q}(\overline{\mathbf{x}})\right) \in \operatorname{im} R_{q, \mathbf{x}}(\overline{\mathbf{x}})$,

(i.c.4) $\mathbf{w}^{T} F_{q^{\prime}}\left(R_{q}(\overline{\mathbf{x}})\right)=0$,

where $\mathbf{w}$ is defined in Proposition 3.2, using $A=R_{q, \mathbf{x}}(\overline{\mathbf{x}})^{T}$ and $\mathbf{b}=H_{\mathbf{x}}(\overline{\mathbf{x}})^{T}$.

Proof. We first prove that each of the sets (i.a), (i.b), (i.c) implies case (i); then we prove the converse.

(i.a): Under (i.a.1) the matrix $R_{q, \mathbf{x}}(\overline{\mathbf{x}})$ is invertible, and $\mathcal{H}^{\prime}$ is $(n-1)$-dimensional and defined by $\left\{\mathbf{x}: H^{\prime}(\mathbf{x}):=H\left(R_{q}^{-1}(\mathbf{x})\right)=0\right\}$. The direction orthogonal to $\mathcal{H}^{\prime}$ at $R_{q}(\overline{\mathbf{x}})$ is therefore given by the nonzero vector $H_{\mathbf{x}}^{\prime}\left(R_{q}(\overline{\mathbf{x}})\right)=H_{\mathbf{x}}(\overline{\mathbf{x}})\left(R_{q, \mathbf{x}}(\overline{\mathbf{x}})\right)^{-1}$, so that (i.a.2) implies the tangency.

(i.b): When $R_{q, \mathbf{x}}(\overline{\mathbf{x}})$ is singular under (i.b.1), we have $\operatorname{dim} \mathcal{H}^{\prime}=n-p$, and the tangent space to $\mathcal{H}^{\prime}$ at $R_{q}(\overline{\mathbf{x}})$ is $\operatorname{im} R_{q, \mathbf{x}}(\overline{\mathbf{x}})$. In fact, given any $\mathbf{u} \in \operatorname{im} R_{q, \mathbf{x}}(\overline{\mathbf{x}})$ and $\mathbf{w}$ such that $\mathbf{u}=R_{q, \mathbf{x}}(\overline{\mathbf{x}}) \mathbf{w}$, there always exist $\mathbf{w}_{\mathcal{H}}$ tangent to $\mathcal{H}$ at $\overline{\mathbf{x}}$ and $\mathbf{z} \in \operatorname{ker} R_{q, \mathbf{x}}(\overline{\mathbf{x}})$ such that $\mathbf{w}=\mathbf{w}_{\mathcal{H}}+\mathbf{z}\left(\operatorname{ker} R_{q, \mathbf{x}}(\overline{\mathbf{x}})\right.$ and $\operatorname{ker} H_{\mathbf{x}}(\overline{\mathbf{x}})$ span $\mathbb{R}^{n}$ by (i.b.1)). Hence, $\mathbf{u}$ is the image of $\mathbf{w}_{\mathcal{H}}$ through $R_{q, \mathbf{x}}(\overline{\mathbf{x}})$; i.e., it is tangent to $\mathcal{H}^{\prime}$ at $R_{q}(\overline{\mathbf{x}})$. Case (i) then follows from (i.b.2).

(i.c): Note that (i.c.2) implies $H_{\mathbf{x}}(\overline{\mathbf{x}})^{T} \in \operatorname{im} R_{q, \mathbf{x}}(\overline{\mathbf{x}})^{T}$ (the null space of a matrix is orthogonal to the image of its transpose); thus by Proposition 3.2 there exists a unique vector $\mathbf{w} \in \operatorname{im} R_{q, \mathbf{x}}(\overline{\mathbf{x}})$ such that $R_{q, \mathbf{x}}(\overline{\mathbf{x}})^{T} \mathbf{w}=H_{\mathbf{x}}(\overline{\mathbf{x}})^{T}$. This implies

$$
\mathbf{w}_{\mathcal{H}}^{T} R_{q, \mathbf{x}}(\overline{\mathbf{x}})^{T} \mathbf{w}=0
$$

for all $\mathbf{w}_{\mathcal{H}}$ tangent to $\mathcal{H}$ at $\overline{\mathbf{x}}$, and

$$
\mathbf{w}_{\mathcal{H}}^{T} R_{q, \mathbf{x}}(\overline{\mathbf{x}})^{T} \mathbf{w}^{\prime} \neq 0
$$

for all $\mathbf{w}^{\prime}$ linearly independent from $\mathbf{w}$ and $\operatorname{in} \operatorname{im} R_{q, \mathbf{x}}(\overline{\mathbf{x}})$; i.e., the tangent space to $\mathcal{H}^{\prime}$ at $R_{q}(\overline{\mathbf{x}})$ is the orthogonal complement of $\mathbf{w}$ in $\operatorname{im} R_{q, \mathbf{x}}$. By (i.c.3) and (i.c.4), it follows that $F_{q^{\prime}}\left(R_{q}(\overline{\mathbf{x}})\right)$ is tangent to $\mathcal{H}^{\prime}$ at $R_{q}(\overline{\mathbf{x}})$.

We can now prove that case (i) implies one and only one of (i.a), (i.b), (i.c). We proceed by partitioning the possible cases into three sets:

(A) $\operatorname{dim} \operatorname{ker} R_{q, \mathbf{x}}(\overline{\mathbf{x}})=0$;

(B) $\operatorname{ker} R_{q, \mathbf{x}}(\overline{\mathbf{x}}) \not \subset$ ker $H_{\mathbf{x}}(\overline{\mathbf{x}})$;

(C) (C.1) dim ker $R_{q, \mathbf{x}}(\overline{\mathbf{x}})>0$, (C.2) $\operatorname{ker} R_{q, \mathbf{x}}(\overline{\mathbf{x}}) \subset \operatorname{ker} H_{\mathbf{x}}(\overline{\mathbf{x}})$.

As we see next, the sets above respectively imply (i.a), (i.b), (i.c), and since the union of (A), (B), (C) covers all possible cases, these implications complete our proof.

(A): The condition is the same as (i.a.1), while (i.a.2) follows by the same argument expressed in the implication (i.a) above.

(B): The condition is the same as (i.b.1). Then, by the same argument expressed in the implication (i.b) above, the tangent space to $\mathcal{H}^{\prime}$ at $R_{q}(\overline{\mathbf{x}})$ is $\operatorname{im} R_{q, \mathbf{x}}(\overline{\mathbf{x}})$, so that (i) implies (i.b.2), hence (i.b).

(C): (C.1) and (C.2) are the same as (i.c.1) and (i.c.2). Then, by the same argument expressed in the implication (i.c) above, the tangent space to $\mathcal{H}^{\prime}$ at $R_{q}(\overline{\mathbf{x}})$ is composed of the 
vectors $\mathbf{w}_{\mathcal{H}^{\prime}} \in \operatorname{im} R_{q, \mathbf{x}}(\overline{\mathbf{x}})$ that are orthogonal to $\mathbf{w}$ in (i.c.4), so that $F_{q^{\prime}}\left(R_{q}(\overline{\mathbf{x}})\right)$ must satisfy (i.c.3) and (i.c.4), hence (i.c).

Lemma 3.4. Case (ii) in Theorem 3.1 is equivalent to the following condition:

(ii) $p_{\mathcal{H}}>0$.

Proof. $\operatorname{dim} \mathcal{H}^{\prime}=n-1-p_{\mathcal{H}}$, whereas $\operatorname{dim} \mathcal{H}=n-1$.

Note that conditions (i.c.1) and (i.c.2) in Lemma 3.3 imply case (ii).

Proof of Theorem 3.1. We first prove that each of the sets (i.a), (i.b), (i.c), and (ii) implies the singularity of $\mathcal{S}$; then we prove that the singularity of $\mathcal{S}$ implies at least one of the sets (i.a), (i.b), (i.c), and (ii).

To simplify the calculations, let us define

$$
\begin{aligned}
A & :=R_{q, \mathbf{x}}(\overline{\mathbf{x}}), \\
\mathbf{u} & :=F_{q^{\prime}}\left(R_{q}(\overline{\mathbf{x}})\right)-R_{q, \mathbf{x}}(\overline{\mathbf{x}}) F_{q}(\overline{\mathbf{x}}), \\
\mathbf{v} & :=\frac{H_{\mathbf{x}}(\overline{\mathbf{x}})^{T}}{H_{\mathbf{x}}(\overline{\mathbf{x}}) F_{q}(\overline{\mathbf{x}})},
\end{aligned}
$$

where $H_{\mathbf{x}}(\overline{\mathbf{x}}) F_{q}(\overline{\mathbf{x}}) \neq 0$ under the hypothesis (H.2). The conditions of Lemmas 3.3 and 3.4 in this notation become

(i.a) (i.a.1) $\operatorname{dim} \operatorname{ker} A=0$,

(i.a.2) $\mathbf{v}^{T} A^{-1} \mathbf{u}=-1$;

(i.b) (i.b.1) $\operatorname{ker} A \not \subset \operatorname{ker} \mathbf{v}^{T}$,

(i.b.2) $\mathbf{u} \in \operatorname{im} A$

(i.c) (i.c.1) $\operatorname{dim} \operatorname{ker} A>0$,

(i.c.2) $\operatorname{ker} A \subset \operatorname{ker} \mathbf{v}^{T}$,

(i.c.3) $\mathbf{u} \in \operatorname{im} A$,

(i.c.4) $\mathbf{v}^{T} A^{\dagger} \mathbf{u}=-1$;

(ii) $\operatorname{dim}\left(\operatorname{ker} A \cap \operatorname{ker} \mathbf{v}^{T}\right)>0$,

while (2.4) becomes

$$
\mathcal{S}:=A+\mathbf{u v}^{T} .
$$

The translation is trivial except for (i.c.4), which we derive as follows. Starting from $\mathbf{w}^{T} F_{q^{\prime}}\left(R_{q}(\overline{\mathbf{x}})\right)$ $=0$, substituting $\mathbf{w}=\left(R_{q, \mathbf{x}}(\overline{\mathbf{x}})^{T}\right)^{\dagger} H_{\mathbf{x}}(\overline{\mathbf{x}})^{T}$, and using (3.1), we obtain

$$
\mathbf{w}^{T} F_{q^{\prime}}\left(R_{q}(\overline{\mathbf{x}})\right)=H_{\mathbf{x}}(\overline{\mathbf{x}}) R_{q, \mathbf{x}}(\overline{\mathbf{x}})^{\dagger} F_{q^{\prime}}\left(R_{q}(\overline{\mathbf{x}})\right) \stackrel{(3.1)}{=} H_{\mathbf{x}}(\overline{\mathbf{x}}) F_{q}(\overline{\mathbf{x}})\left(\mathbf{v}^{T} A^{\dagger} \mathbf{u}+\mathbf{v}^{T} A^{\dagger} A F_{q}(\overline{\mathbf{x}})\right)=0 .
$$

Then, noting that $A^{\dagger} A$ is an orthogonal projector onto $\operatorname{im} A^{T}$, i.e., $A^{\dagger} A F_{q}(\overline{\mathbf{x}})=F_{q}(\overline{\mathbf{x}})-\mathbf{z}$, where $\mathbf{z}$ is the component of $F_{q}(\overline{\mathbf{x}})$ in ker $A$, and using (3.1) once again, we obtain

$$
\mathbf{v}^{T} A^{\dagger} \mathbf{u}+\mathbf{v}^{T} A^{\dagger} A F_{q}(\overline{\mathbf{x}}) \stackrel{(3.1), A^{\dagger} A F_{q} \in \operatorname{im} A^{T}}{=} \mathbf{v}^{T} A^{\dagger} \mathbf{u}+1-\mathbf{v}^{T} \mathbf{z}=0
$$

where we have dropped the term $H_{\mathbf{x}}(\overline{\mathbf{x}}) F_{q}(\overline{\mathbf{x}})$ by (H.2). The translation is completed by noting that, by (i.c.2), we have $\mathbf{v}^{T} \mathbf{z}=0$.

(i.a): (i.a.2) implies $\mathbf{u} \neq \mathbf{0}$. Hence, the vector $\mathbf{z}:=A^{-1} \mathbf{u}$ is well defined and nonzero, and, using again (i.a.2), we have

$$
\mathcal{S} \mathbf{z}=A A^{-1} \mathbf{u}+\mathbf{u v}^{T} A^{-1} \mathbf{u}=\mathbf{0}
$$


i.e., $\mathbf{z} \in \operatorname{ker} \mathcal{S}$ and $\mathcal{S}$ is singular.

(i.b): Condition (i.b.1) implies $\operatorname{dim} \operatorname{ker} A>0$. If $\mathbf{u}=\mathbf{0}$, then $\mathcal{S}=A$ is singular. Otherwise, by (i.b.2) and Proposition 3.2 there exists a unique $\mathbf{w} \in \operatorname{im} A^{T}$ (i.e., orthogonal to $\operatorname{ker} A$ ) such that $A \mathbf{w}=\mathbf{u}$. By (i.b.1) we can take $\mathbf{z} \in \operatorname{ker} A, \mathbf{z} \notin \operatorname{ker} \mathbf{v}^{T}$ such that $\mathbf{v}^{T}\left(\mathbf{w}^{\prime}+\mathbf{z}\right)=-1$. We have $\mathcal{S}(\mathbf{w}+\mathbf{z})=A \mathbf{w}+\mathbf{u v}^{T}(\mathbf{w}+\mathbf{z})=\mathbf{u}-\mathbf{u}=\mathbf{0}$; i.e., $(\mathbf{w}+\mathbf{z}) \in \operatorname{ker} \mathcal{S}$.

(i.c): If $\mathbf{u}=\mathbf{0}$, then $\mathcal{S}=A$ is singular by (i.c.1), with $\operatorname{ker} \mathcal{S}=\operatorname{ker} A$. Otherwise, by (i.c.3) and Proposition 3.2 there exists $\mathbf{w}=A^{\dagger} \mathbf{u}$. We have $\mathcal{S} \mathbf{w}=A A^{\dagger} \mathbf{u}+\mathbf{u v}^{T} \mathbf{A}^{\dagger} \mathbf{u}$; using (i.c.3), (i.c.4), and the fact that $A A^{\dagger}$ is an orthogonal projector onto $\operatorname{im} A, \mathcal{S} \mathbf{w}=\mathbf{u}-\mathbf{u}=0$; i.e., $\mathbf{w} \in \operatorname{ker} \mathcal{S}$

(ii): If $\mathbf{u}=\mathbf{0}$, then by (2.4), $\mathcal{S}=A$ is singular with $\operatorname{ker} \mathcal{S}=\operatorname{ker} A$. Otherwise, the implication follows from (ii) by taking $\mathbf{z} \in \operatorname{ker} A \cap \operatorname{ker} \mathbf{v}^{T}$.

We can now prove that $\operatorname{dim} \operatorname{ker} \mathcal{S}>0$ implies at least one of the sets (i.a), (i.b), (i.c), and (ii). We proceed by partitioning the possible cases into four sets:

(I) (I.1) $\operatorname{dim} \operatorname{ker} A=0$;

(II) (II.1) $\operatorname{dim} \operatorname{ker} A>0$,

(II.2) $\operatorname{dim}(\operatorname{ker} A \cap \operatorname{ker} \mathcal{S})=0$,

(II.3) $\mathbf{u} \neq \mathbf{0}$

(III) (III.1) $\operatorname{dim}(\operatorname{ker} A \cap \operatorname{ker} \mathcal{S})>0$,

(III.2) $\mathbf{u} \neq \mathbf{0}$

(IV) (IV.1) $\operatorname{dim} \operatorname{ker} A>0$,

$(\mathrm{IV} .2) \mathbf{u}=\mathbf{0}$.

As we see next, each of the sets above implies at least one of (i.a), (i.b), (i.c), (ii), and since the union of (I)-(IV) covers all possible cases, these implications complete our proof.

(I): (I.1) is equal to (i.a.1). Together with $\operatorname{dim} \operatorname{ker} \mathcal{S}>0$, they imply the existence of $\mathbf{z} \neq \mathbf{0}$ such that

$$
A \mathbf{z}+\mathbf{u v}^{T} \mathbf{z}=\mathbf{0}, \quad \text { with } \quad \mathbf{v}^{T} \mathbf{z} \neq 0 .
$$

Left-multiplying the above equation by $\mathbf{v}^{T} A^{-1}$, we obtain

$$
\mathbf{v}^{T} \mathbf{z}+\left(\mathbf{v}^{T} A^{-1} \mathbf{u}\right) \mathbf{v}^{T} \mathbf{z}=0
$$

which implies (i.a.2) and hence (i.a).

(II): By (II.1) and (II.2), given $\mathbf{0} \neq \mathbf{z} \in \operatorname{ker} A$, we have $\mathcal{S} \mathbf{z}=\mathbf{u v}^{T} \mathbf{z} \neq 0$, so that $\mathbf{u} \neq \mathbf{0}$ ((II.3) is superfluous) and $\mathbf{v}^{T} \mathbf{z} \neq 0$, the latter being equivalent to (i.b.1). Moreover, taking $\mathbf{0} \neq \mathbf{z} \in \operatorname{ker} \mathcal{S}$, we have $\mathcal{S} \mathbf{z}=A \mathbf{z}+\mathbf{u v}^{T} \mathbf{z}=\mathbf{0}$ with $\mathbf{0} \neq A \mathbf{z} \in \operatorname{im} A$ by (II.2), so that the latter equation implies (i.b.2), hence (i.b).

(III): By (III.1), given $\mathbf{0} \neq \mathbf{z} \in(\operatorname{ker} A \cap \operatorname{ker} \mathcal{S})$, we have $\mathcal{S} \mathbf{z}=\mathbf{u v}^{T} \mathbf{z}=\mathbf{0}$, with $\mathbf{u} \neq \mathbf{0}$ by (III.2), so that we have $\mathbf{v}^{T} \mathbf{z}=0$, which implies case (ii).

(IV): (IV.2) means that

$$
F_{q^{\prime}}\left(R_{q}(\overline{\mathbf{x}})\right)=A F_{q}(\overline{\mathbf{x}})
$$

Let us consider separately the case where $F_{q^{\prime}}\left(R_{q}(\overline{\mathbf{x}})\right)$ is tangent to $\mathcal{H}^{\prime}$ at $R_{q}(\overline{\mathbf{x}})$ (including the case $\left.F_{q^{\prime}}\left(R_{q}(\overline{\mathbf{x}})\right)=\mathbf{0}\right)$, and the case where $F_{q^{\prime}}\left(R_{q}(\overline{\mathbf{x}})\right) \neq \mathbf{0}$ is transverse to $\mathcal{H}^{\prime}$ at $R_{q}(\overline{\mathbf{x}})$. In the first case we have (3.2) together with $F_{q^{\prime}}\left(R_{q}(\overline{\mathbf{x}})\right)=A \mathbf{w}_{\mathcal{H}}$, with $\mathbf{w}_{\mathcal{H}}$ tangent to $\mathcal{H}$ 
Table 1

Each row describes a possible combination of the cases in Lemmas 3.3 and 3.4, distinguishing $\mathbf{u}=0$ from $\mathbf{u} \neq 0$. The dimension $s$ and a base of $\operatorname{ker} \mathcal{S}$ are reported. Notation: $p:=\operatorname{dim} \operatorname{ker} R_{q, \mathbf{x}}(\overline{\mathbf{x}})$ is $\geq 1$ whenever used $(p \geq 2$ in rows 3 and 5$) ; p_{\mathcal{H}}:=\operatorname{dim}\left(\operatorname{ker} R_{q, \mathbf{x}}(\overline{\mathbf{x}}) \cap \operatorname{ker} H_{\mathbf{x}}(\overline{\mathbf{x}})\right) ; \mathbf{z}_{1}, \ldots, \mathbf{z}_{p}$ is a base of $\operatorname{ker} R_{q, \mathbf{x}}(\overline{\mathbf{x}})$, with $\mathbf{z}_{1}, \ldots, \mathbf{z}_{p-1} \in \operatorname{ker} H_{\mathbf{x}}(\overline{\mathbf{x}}) ; \mathbf{u}:=F_{q^{\prime}}\left(R_{q}(\overline{\mathbf{x}})\right)-R_{q, \mathbf{x}}(\overline{\mathbf{x}}) F_{q}(\overline{\mathbf{x}}) ; \mathbf{z}:=\left(H_{\mathbf{x}}(\overline{\mathbf{x}}) R_{q, \mathbf{x}}(\overline{\mathbf{x}})^{\dagger} \mathbf{u}+H_{\mathbf{x}}(\overline{\mathbf{x}}) F_{q}(\overline{\mathbf{x}})\right) /\left(H_{\mathbf{x}}(\overline{\mathbf{x}}) \mathbf{z}_{p}\right) \mathbf{z}_{p}$.

\begin{tabular}{|c|c|c|c|c|c|c|}
\hline (i.a) & (i.b) & (i.c) & (ii) & $\mathbf{u}=\mathbf{0}$ & $s$ & $\operatorname{ker} \mathcal{S}$ \\
\hline \multirow[t]{9}{*}{$\checkmark$} & & & & & 1 & $R_{q, \mathbf{x}}(\overline{\mathbf{x}})^{-1} \mathbf{u}$ \\
\hline & $\checkmark$ & & & & 1 & $R_{q, \mathbf{x}}(\overline{\mathbf{x}})^{\dagger} \mathbf{u}-\mathbf{z}$ \\
\hline & $\checkmark$ & & $\checkmark$ & & $p$ & $\mathbf{z}_{1}, \ldots, \mathbf{z}_{p-1}, R_{q, \mathbf{x}}(\overline{\mathbf{x}})^{\dagger} \mathbf{u}-\mathbf{z}$ \\
\hline & $\checkmark$ & & & $\checkmark$ & 1 & $\mathbf{z}_{1}$ \\
\hline & $\checkmark$ & & $\checkmark$ & $\checkmark$ & $p$ & $\mathbf{z}_{1}, \ldots, \mathbf{z}_{p}$ \\
\hline & & $\checkmark$ & $\checkmark$ & & $p+1$ & $\mathbf{z}_{1}, \ldots, \mathbf{z}_{p}, R_{q, \mathbf{x}}(\overline{\mathbf{x}})^{\dagger} \mathbf{u}$ \\
\hline & & $\checkmark$ & $\checkmark$ & $\checkmark$ & $p$ & $\mathbf{z}_{1}, \ldots, \mathbf{z}_{p}$ \\
\hline & & & $\checkmark$ & & $p_{\mathcal{H}}$ & $\mathbf{z}_{1}, \ldots, \mathbf{z}_{p_{\mathcal{H}}}$ \\
\hline & & & $\checkmark$ & $\checkmark$ & $p$ & $\mathbf{z}_{1}, \ldots, \mathbf{z}_{p}$ \\
\hline
\end{tabular}

at $\overline{\mathbf{x}}$; therefore $0=A\left(F_{q}(\overline{\mathbf{x}})-\mathbf{w}_{\mathcal{H}}\right)$; i.e, $F_{q}(\overline{\mathbf{x}})-\mathbf{w}_{\mathcal{H}} \in \operatorname{ker} A$. By the hypothesis (H.2), $F_{q}(\overline{\mathbf{x}})-\mathbf{w}_{\mathcal{H}} \notin \operatorname{ker} \mathbf{v}$, i.e., (i.b.1), whereas (i.b.2) is trivially satisfied by (3.2), hence (i.b). In the second case, $F_{q^{\prime}}\left(R_{q}(\overline{\mathbf{x}})\right) \in \operatorname{im} A$ is not tangent to $\mathcal{H}^{\prime}$ at $R_{q}(\overline{\mathbf{x}})$, so that we have (i.c.1) (by (IV.1)) and (i.c.2) (otherwise $\operatorname{im} A$ would be all tangent to $\mathcal{H}^{\prime}$ at $R_{q}(\overline{\mathbf{x}})$ ). Conditions (i.c.1) and (i.c.2) imply (ii).

Corollary 3.5. The rank defect $s$ of $\mathcal{S}$ and a base of ker $\mathcal{S}$ for each case in Lemmas 3.3 and 3.4 are as shown in Table 1.

Proof. Condition (i.a.1) excludes (i.b), (i.c.), (ii), and $\mathbf{u}=0$, and the first row of the table follows from the computation of $\operatorname{ker} \mathcal{S}$ in the implication (i.a) in the proof of Theorem 3.1.

Rows 4, 5, 7, 9 pertain to the case $\mathbf{u}=0$. Here $\mathcal{S}=R_{q, \mathbf{x}}(\overline{\mathbf{x}})$, and regardless of which set of conditions is satisfied, we have that $\operatorname{ker} \mathcal{S}=\operatorname{ker} R_{q, \mathbf{x}}(\overline{\mathbf{x}})$.

Let's now restrict our attention to the cases $\mathbf{u} \neq 0$. (i.b) can occur together with (ii), or alone. If (i.b) and not (ii) (row 2), then from the proof of Theorem 3.1 (implication (i.b)), we have that ker $\mathcal{S}$ is one-dimensional and spanned by vector $\mathbf{w}+\mathbf{z}$, where $\mathbf{z} \in \operatorname{ker} A$ is such that $\mathbf{v}^{T}(\mathbf{w}+\mathbf{z})=-1$. Imposing this condition and letting $\mathbf{z}:=\zeta \mathbf{z}_{p}$, we obtain $\zeta=-\left(\mathbf{v}^{T} \mathbf{w}_{1}\right) /\left(\mathbf{v}^{T} \mathbf{z}_{p}\right)$. Substituting (3.1) into this expression, we obtain the expression of $\mathbf{z}$ in the caption of Table 1.

If (i.b) and (ii) (row 3), the vector $\mathbf{w}+\mathbf{z}$ found above together with all vectors in $\operatorname{ker} R_{q, \mathbf{x}}(\overline{\mathbf{x}}) \cap \operatorname{ker} H_{\mathbf{x}}(\mathbf{x})$ form a base of $\operatorname{ker} \mathcal{S}$.

Case (i.c) (row 6) always implies (ii). From the proof of Theorem 3.1 (implication (i.c)), we have that $\operatorname{ker} \mathcal{S}$ is spanned by a base of $\operatorname{ker} R_{q, \mathbf{x}}(\overline{\mathbf{x}})$ and by vector $\mathbf{w}$.

Condition (ii) may also occur alone (row 8) when $F_{q^{\prime}}\left(R_{q}(\overline{\mathbf{x}})\right)$ is not tangent to $\mathcal{H}^{\prime}$ at $R_{q}(\overline{\mathbf{x}})$. In this case $\operatorname{ker} \mathcal{S}=\operatorname{ker} R_{q, \mathbf{x}}(\overline{\mathbf{x}}) \cap \operatorname{ker} H_{\mathbf{x}}(\overline{\mathbf{x}})$.

Corollary 3.6. Let $R_{q}^{\prime}$ and $R_{q}^{\prime \prime}$ be two different smooth extensions of the same reset map $R_{q}$ to an open neighborhood of $\mathbf{x} \in G_{q}$. The ranks of the saltation matrices based on $R_{q}^{\prime}$ and $R_{q}^{\prime \prime}$ at $\mathbf{x}$ are the same.

In other words, as we expected, the rank property of the saltation matrix depends on the properties of $R_{q}: G_{q} \rightarrow X$ but not of its smooth extension, even though the algebraic 
formula of the saltation matrix uses the differential $R_{q, \mathbf{x}}(\mathbf{x})$ and therefore requires the choice of a smooth extension of $R_{q}$ to an open neighborhood in $X$.

Finally, we make a remark about the case $F_{q^{\prime}}\left(R_{q}(\overline{\mathbf{x}})\right)=\mathbf{0}$.

Corollary 3.7. If $R_{q}(\overline{\mathbf{x}})$ is an equilibrium of the vector field $F_{q^{\prime}}$, then the matrix $\mathcal{S}$ in (2.4) is singular.

4. The special case of the Filippov systems. Case (i.a) in Theorem 3.1 is particularly significant for a special class of discontinuous systems known as the Filippov (or piecewise smooth) systems [33]. A Filippov system is a collection of vector fields $F_{q}$ defined on domains $D_{q} \subset \mathbb{R}^{n}$ separated by a set of (possibly intersecting) $(n-1)$-dimensional smooth manifolds. A solution of a Filippov system is any absolutely continuous function that satisfies almost everywhere the differential inclusion

$$
\dot{\mathbf{x}} \in \operatorname{conv}\left(F_{q_{1}}(\mathbf{x}), \ldots, F_{q_{n}}(\mathbf{x})\right),
$$

where conv is the convex hull and $D_{q_{1}}, \ldots, D_{q_{n}}$ is the set of domains that intersect every arbitrary small neighborhood of $\mathbf{x}$. By the above definition, a given initial condition $\mathbf{x}$ may admit multiple solutions, whenever the differential inclusion (4.1) admits multiple trajectories starting at $\mathbf{x}$, and solutions may belong to the interior of a domain or they may slide along a boundary between two or more domains when this is consistent with (4.1). More precisely, if the convex hull of $F_{q_{1}}, \ldots, F_{q_{n}}$ has no intersection with the tangent space to the boundary, the only admissible solution of (4.1) is one that crosses the boundary. If the convex hull intersects the tangent space to the boundary, but all vector fields locally point out of their respective domains, then locally solutions of (4.1) are bound to slide on the boundary. If the convex hull intersects the tangent space to the boundary, and some of the vector fields locally point inside their domain, then (4.1) admits both solutions that slide along the boundary and solutions that leave the boundary and enter one of the neighboring domains. Discontinuity boundaries are consequently partitioned into crossing, sliding, and escaping regions.

In light of Definition 2.1, Filippov systems are a particular type of hybrid systems, where a value of the discrete variable $q$ is assigned to each of the smooth domains of the Filippov system as well as to each of the sliding regions. For each $q$, the discontinuity set $G_{q}$ collects all the discontinuity manifolds triggering a switch of the vector field; e.g., the manifold where one of the hybrid-flows is tangent to a discontinuity manifold marks the exit from a sliding region, thus triggering the switch from a sliding to a regular vector field. The switch is defined by the reset map $\left(R_{q}(\overline{\mathbf{x}}), q^{\prime}\right)=R(\overline{\mathbf{x}}, q)$, whose $X$-component $R_{q}$ is always the identity. The existence of multiple solutions calls for a more general notion of trajectory than the one given in Definition 2.2, but as long as we keep away from the escaping regions, Definition 2.2 can be applied.

From Theorem 3.1 (case (i)) and Corollary 3.5 we have the following. ${ }^{5}$

Corollary 4.1. Given a Filippov system with unique forward-time solutions, the saltation matrix upon entering at $\overline{\mathbf{x}}$ an $(n-1)$-dimensional sliding region is singular, with rank defect 1 and null space spanned by $F_{1}(\overline{\mathbf{x}})-F_{2}(\overline{\mathbf{x}}), F_{1}$ and $F_{2}$ being the vector fields defined on the two sides of the sliding region. The saltation matrix upon exiting the sliding region is the identity.

\footnotetext{
${ }^{5}$ Hypotheses (H.0)-(H.2) are assumed to hold in a neighborhood of the system's reference trajectory.
} 

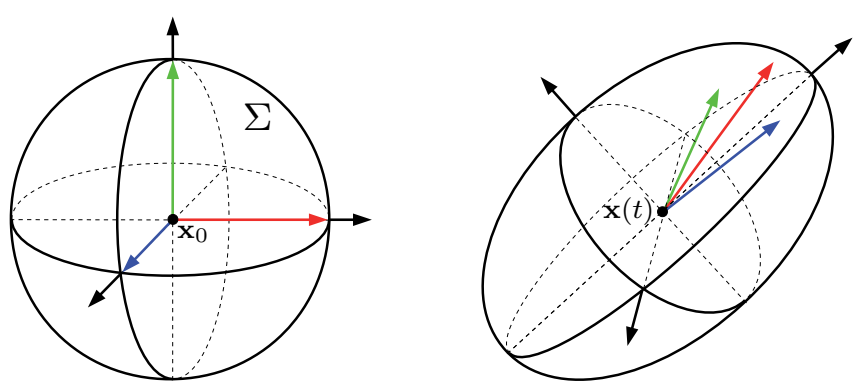

Figure 1. A three-dimensional example of the first-order deformation of the sphere $\Sigma$ (left) into an ellipsoid (right). The vectors of a generic base of $\mathbb{R}^{3}$ tend to align, as $t \rightarrow \infty$, with the principal axis of the ellipsoid.

Proof. The first part of the statement follows by applying Theorem 3.1 (case (i)), and Corollary 3.5. For the second part, note that on exiting sliding, we have $F_{q^{\prime}}\left(R_{q}(\overline{\mathbf{x}})\right)-$ $R_{q, \mathbf{x}}(\overline{\mathbf{x}}) F_{q}(\overline{\mathbf{x}})=0$ in $(2.4)$.

Our analysis extends some known results obtained by Dieci and Lopez in 2011 [30] for the case of Filippov systems. Case (i.a) of Theorem 3.1 generalizes their Lemma 2.3. Moreover, Theorem 2.8 in [30] states that if a trajectory satisfying our hypotheses (H.1) and (H.2) encounters several distinct $(n-1)$-dimensional sliding regions, the resulting fundamental solution matrix generically has rank defect 1 . This directly follows from our Proposition 2.4 and Corollary 4.1.

Another interesting case occurs when a trajectory, already sliding on an $(n-1)$-dimensional discontinuity manifold $\mathcal{H}_{1}$ along the vector field $F_{q}$, reaches the intersection between two such manifolds at some $\overline{\mathbf{x}}$ and begins sliding in the $(n-2)$-dimensional intersection along the vector field $F_{q^{\prime}}$ (see "From one surface to the intersection" on page 945 in [30]). According to Corollary 4.1, the null space of the saltation matrix $\mathcal{S}$ encountered at $\overline{\mathbf{x}}$ is spanned by $F_{q^{\prime}}(\overline{\mathbf{x}})-F_{q}(\overline{\mathbf{x}})$, which is tangent to $\mathcal{H}_{1}$ (both vectors $F_{q^{\prime}}(\overline{\mathbf{x}})$ and $F_{q}(\overline{\mathbf{x}})$ are tangent to $\mathcal{H}_{1}$ at $\overline{\mathbf{x}})$. If $\operatorname{dim} \operatorname{ker} \psi^{-}\left(\bar{t}, t_{0}\right)=1$, then $\operatorname{im} \psi^{-}\left(\bar{t}, t_{0}\right)$ can be shown to be the tangent space to $\mathcal{H}_{1}$ at $\overline{\mathbf{x}}$. Therefore we have $\operatorname{ker} \mathcal{S} \subset \operatorname{im} \psi^{-}\left(\bar{t}, t_{0}\right)$, so that according to Proposition 2.3, $\operatorname{dim} \operatorname{ker} \psi^{+}\left(\bar{t}, t_{0}\right)=2$. This is an example where the transversality assumption of Proposition 2.4 is broken.

5. Numerical computation of the Lyapunov exponents in a hybrid system. Let us first review the concept of Lyapunov exponents (LEs) in a smooth system such as (2.1). Consider a sphere $\Sigma$ centered at $\mathbf{x}_{0}$ (see Figure 1 (left)). The image of any point $\mathbf{x}^{\prime}\left(t_{0}\right) \in \Sigma$ under the flow at some $t>t_{0}$ is given to first order by

$$
\mathbf{x}^{\prime}(t)=\mathbf{x}(t)+\psi\left(t, t_{0}\right)\left(\mathbf{x}^{\prime}\left(t_{0}\right)-\mathbf{x}_{0}\right),
$$

where $\psi\left(t, t_{0}\right)$ is the fundamental solution matrix defined by (2.2). Since the above system is linear, the sphere is mapped to first order onto an ellipsoid (see Figure 1 (right)). Assuming the radius of the sphere at time $t_{0}$ to be 1 , the lengths of the principal axes of the ellipsoid at time $t$ are the singular values $\sigma_{1}(t) \geq \sigma_{2}(t) \geq \cdots \geq \sigma_{n}(t)$ of the matrix $\psi\left(t, t_{0}\right)$. The $i$ th $\operatorname{LE} \lambda_{i}$ of the reference trajectory $\mathbf{x}(t)$ measures the average exponential growth of the $i$ th principal 
axis of the ellipsoid:

$$
\lambda_{i}:=\lim _{t \rightarrow \infty} \frac{1}{t} \ln \sigma_{i}(t), \quad i=1, \ldots, n .
$$

The computation of the LEs is based on the computation of the fundamental solution matrix $\psi\left(t, t_{0}\right)$. The most popular numerical methods are variations of the discrete QR algorithm $[5,6,52,48]$, which we briefly recall here in continuous time. The idea is to work on a set $\mathcal{T}$ of time instants $\left\{t_{0}, t_{1}, t_{2}, \ldots\right\}, t_{j}>t_{j-1}$, and compute the fundamental solution matrix $\psi\left(t, t_{j-1}\right)$ in each time interval $\left[t_{j-1}, t_{j}\right], j \geq 1$. This is done by simulating the linearized dynamics $\dot{\psi}^{(j)}\left(t, t_{j-1}\right)=J(\mathbf{x}(t)) \psi^{(j)}\left(t, t_{j-1}\right)$ starting from the initial condition $\psi^{(j)}\left(t_{j-1}, t_{j-1}\right)=$ $Q^{(j-1)}$ obtained for $j \geq 2$ from the decomposition $\psi^{(j-1)}\left(t_{j-1}, t_{j-2}\right)=Q^{(j-1)} R^{(j-1)}$, with $Q^{(0)}=R^{(0)}=I$. Then, by the initial condition $\psi\left(t_{j-1}, t_{j-1}\right)=I$ used in the definition (2.3), we obtain $\psi\left(t, t_{j-1}\right) Q^{(j-1)}=\psi^{(j)}\left(t, t_{j-1}\right)$ for $t \in\left[t_{j-1}, t_{j}\right]$, and the composition of the fundamental solution matrices gives

$$
\psi\left(t_{j}, t_{0}\right)=\prod_{k=1}^{j} \psi\left(t_{j-k+1}, t_{j-k}\right)=Q^{(j)} \prod_{k=1}^{j} R^{(j-k+1)} .
$$

The $n$ LEs associated to the reference trajectory $\mathbf{x}(t)$ are approximated by

$$
\lambda_{i}=\lim _{j \rightarrow \infty} \frac{1}{t_{j}-t_{0}} \sum_{k=1}^{j} \ln \left|R_{i, i}^{(k)}\right| .
$$

Accurately performing QR decomposition (typically resorting to the Gram-Schmidt algorithm) is extremely important from a numerical standpoint, since the magnitude of each of the columns of $\psi\left(t, t_{0}\right)$ tends to diverge/vanish if the column is associated to a positive/negative LE [52]. The repeated reorthonormalization of the fundamental matrix allows us to avoid numerical cancellations.

Let us now focus on the case of a hybrid system (under hypotheses (H.0)-(H.2)). Consider the first singularity reached by the reference trajectory $\mathbf{x}(t)$ at time $t=\bar{t} \in\left[t_{j-1}, t_{j}\right]$; i.e., a singular saltation matrix $\mathcal{S}$ with $\operatorname{dim} \operatorname{ker} \mathcal{S}=s>0$ is encountered at time $t=\bar{t}$, the rank of $\psi^{(j)}\left(\bar{t}, t_{j-1}\right)$ is $n$, and $\operatorname{dim} \operatorname{ker} \mathcal{S} \psi^{(j)}\left(\bar{t}, t_{j-1}\right)=s$ according to Proposition 2.3. We then insert $\bar{t}$ as the $j$ th time instant in the set $\mathcal{T}$ and evaluate the $\mathrm{QR}$ decomposition of the singular matrix $\mathcal{S} \psi^{(j)}\left(\bar{t}, t_{j-1}\right)$. This produces an upper triangular matrix $\bar{R}$ with a bottom-right $s \times s$ block of null elements and a matrix $\bar{Q}=\left[\overline{\mathbf{q}}_{1}|\cdots| \overline{\mathbf{q}}_{n-s} \mid \cdots\right]$ with the first $n-s$ columns orthonormal. The LEs corresponding to the $s$ null elements of the diagonal of $\bar{R}$ are set to $-\infty$, whereas only the $n-s$ columns $\left[\overline{\mathbf{q}}_{1}|\cdots| \overline{\mathbf{q}}_{n-s}\right]$ of $\bar{Q}$ (those corresponding to nonzero diagonal elements of $\bar{R}$ ) are used to initialize the following reduced variational problem:

$$
\begin{aligned}
\dot{\psi}_{R}^{(j+1)}(t, \bar{t}) & =J(\mathbf{x}(t)) \psi_{R}^{(j+1)}(t, \bar{t}), \\
\psi_{R}^{(j+1)}(\bar{t}, \bar{t}) & =\left[\overline{\mathbf{q}}_{1}|\cdots| \overline{\mathbf{q}}_{n-s}\right],
\end{aligned}
$$

where $\psi_{R}^{(j+1)}(t, \bar{t})$ is an $n \times(n-s)$ matrix. The computation of the remaining $n-s$ LEs can now be restarted using the solution of (5.3) along the trajectory $\mathbf{x}(t)$ (analogously to what has 
been done in the literature on DAEs; see, e.g., [43, 44]). Further intersections of discontinuity sets characterized by singular saltation matrices may induce further losses in the dimension of the ellipsoid. When this happens, the dimension of the reduced variational system is adjusted accordingly.

It is worth remarking that, in a hybrid system, the LEs computed along a trajectory do not necessarily coincide with those of the attractor reached by the trajectory. This is a well-known property of some atypical trajectories in a smooth system (consider, e.g., the stable manifolds of the infinitely many saddle cycles embedded in chaotic attractors), but in a hybrid system the phenomenon can happen for typical trajectories, that is, starting from open sets of initial conditions within the basin of attraction. Indeed, even if the attractor does not intersect any discontinuity set, trajectories from further away in the basin of attraction can encounter discontinuities. Some of the LEs of these trajectories may then be at $-\infty$, while the others coincide with some of the attractor's LEs. An example of this is presented in section 6.1, where the LEs of trajectories converging to a three-dimensional chaotic attractor lose, due to a discontinuity, the finite negative exponent of the attractor.

\section{Examples.}

6.1. Tritrophic food chain with conditional harvesting of the superpredator. We consider a tritrophic food chain model [35,3]. The quantities $x_{1}, x_{2}$, and $x_{3}$ represent the abundances of the prey, predator, and superpredator, respectively. The dynamics of the food chain in the absence of harvesting is governed by the equations

$$
F_{1}(\mathbf{x}):=\left(\begin{array}{c}
r x_{1}\left(1-\frac{x_{1}}{K}\right)-\frac{a_{1} x_{1} x_{2}}{1+a_{1} b_{1} x_{1}} \\
e_{1} \frac{a_{1} x_{1} x_{2}}{1+a_{1} b_{1} x_{1}}-m_{1} x_{2}-\frac{a_{2} x_{2} x_{3}}{1+a_{2} b_{2} x_{2}} \\
e_{2} \frac{a_{2} x_{2} x_{3}}{1+a_{2} b_{2} x_{2}}-m_{2} x_{3}
\end{array}\right) .
$$

We assume that harvesting is allowed when the superpredator abundance is above a threshold $\bar{x}_{3}$. This introduces an additional term $-\Gamma x_{3}$ in the equation of $\dot{x}_{3}$ when $x_{3}>\bar{x}_{3}$.

Such a system is typically modelled as a Filippov system, with the vector field above threshold given by

$$
F_{2}\left(x_{1}, x_{2}, x_{3}\right):=F_{1}\left(x_{1}, x_{2}, x_{3}\right)+\left(\begin{array}{c}
0 \\
0 \\
-\Gamma x_{3}
\end{array}\right) .
$$

It is easy to show that sliding is always possible on the manifold $\mathcal{H}:=\left\{\mathbf{x}: x_{3}-\bar{x}_{3}=0\right\}$, provided that $\mathbf{x} \in \mathcal{H}$ belongs to the set $\mathcal{A}:=\left\{\mathbf{x}: F_{1,3}(\mathbf{x})>0\right.$ and $\left.F_{2,3}(\mathbf{x})<0\right\}, F_{i, j}$ being the $j$ th component of $F_{i}$; i.e., the sliding region is the set $\{\mathbf{x}: \mathbf{x} \in \mathcal{H} \cap \mathcal{A}\}$. The flow crosses from below $\mathcal{H}$ in $\left\{\mathbf{x}: \mathbf{x} \in \mathcal{H} \backslash \mathcal{A}, F_{1,3}(\mathbf{x})>0\right\}$ and crosses from above $\mathcal{H}$ in $\left\{\mathbf{x}: \mathbf{x} \in \mathcal{H} \backslash \mathcal{A}, F_{1,3}(\mathbf{x})<0\right\}$.

We can write the model as a hybrid system using three domains, describing dynamics below threshold (domain 1), above threshold (domain 2), and sliding (domain 3). In domain 1, the vector field is (6.1), the discontinuity set is $G_{1}=\left\{\mathbf{x}: \mathbf{x} \in \mathcal{H}, F_{1,3}(\mathbf{x})>0\right\}$, and the reset 


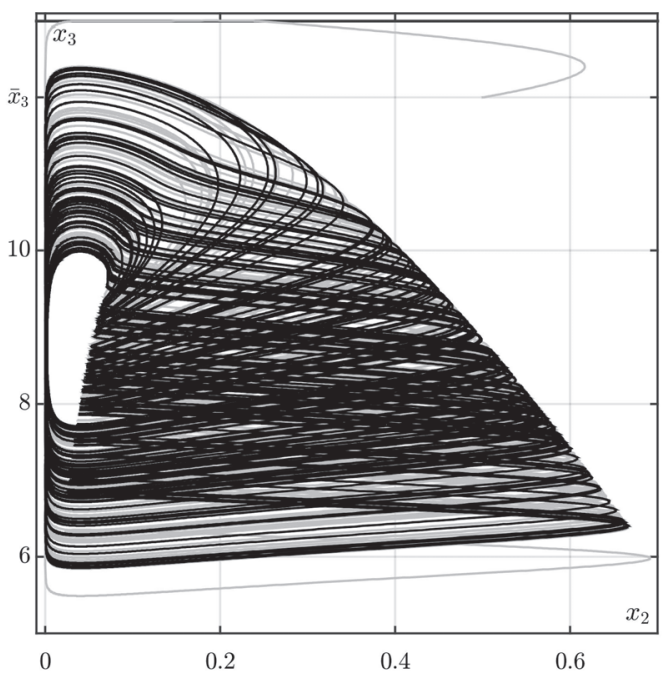

(a)

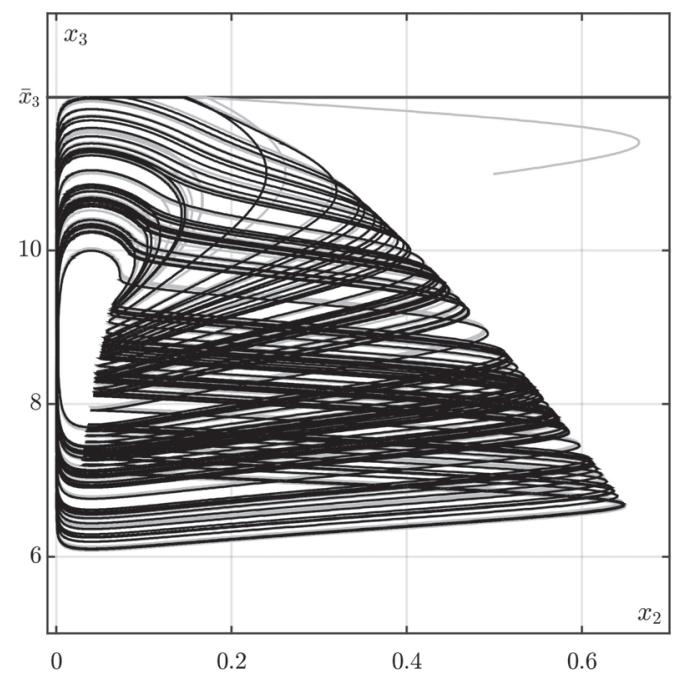

(b)

Figure 2. A projection on the $\left(x_{2}, x_{3}\right)$ plane of the chaotic attractor exhibited by the tritrophic food chain model with conditional harvesting of the superpredator. (a) $\Gamma=0.01, \bar{x}_{3}=13$, the attractor is internal to region 1 with no harvesting. (b) $\Gamma=0.01, \bar{x}_{3}=12$, the attractor involves repeated sliding. Other parameters as in (6.3).

map is $R(\mathbf{x}, 1)=(\mathbf{x}, 2)$ when $\mathbf{x} \in \mathcal{H} \backslash \mathcal{A}, R(\mathbf{x}, 1)=(\mathbf{x}, 3)$ otherwise. In domain 2 , the vector field is (6.2), the discontinuity set is $G_{2}=\left\{\mathbf{x}: \mathbf{x} \in \mathcal{H}, F_{2,3}(\mathbf{x})<0\right\}$, and the reset map is $R(\mathbf{x}, 2)=(\mathbf{x}, 1)$ when $\mathbf{x} \in \mathcal{H} \backslash \mathcal{A}$, and $R(\mathbf{x}, 2)=(\mathbf{x}, 3)$ otherwise. When the system is sliding (domain 3 ), the vector field is

$$
F_{3}\left(x_{1}, x_{2}, x_{3}\right):=\left(\begin{array}{c}
r x_{1}\left(1-\frac{x_{1}}{K}\right)-\frac{a_{1} x_{1} x_{2}}{1+a_{1} b_{1} x_{1}} \\
e_{1} \frac{a_{1} x_{1} x_{2}}{1+a_{1} b_{1} x_{1}}-m_{1} x_{2}-\frac{a_{2} x_{2} x_{3}}{1+a_{2} b_{2} x_{2}} \\
0
\end{array}\right),
$$

the discontinuity set is $G_{3}=\left\{\mathbf{x}: F_{1,3}(\mathbf{x})=0\right.$ or $\left.F_{2,3}(\mathbf{x})=0\right\}$, and the reset map is $R(\mathbf{x}, 3)=$ $(\mathbf{x}, 1)$ when $F_{1,3}(\mathbf{x})=0$, and $R(\mathbf{x}, 3)=(\mathbf{x}, 2)$ otherwise.

We set the system parameters as in Belykh, Piccardi, and Rinaldi [3], i.e.,

$$
\begin{aligned}
& r=1.15, \quad K=1.07 \text {, } \\
& a_{1}=5, \quad b_{1}=0.6, \quad m_{1}=0.4, \quad e_{1}=1, \\
& a_{2}=0.1, \quad b_{2}=20, \quad m_{2}=0.0037, \quad e_{2}=1 \text {, }
\end{aligned}
$$

and use harvesting $\Gamma=0.01$. If the harvesting threshold $\bar{x}_{3}$ is sufficiently high (e.g., $\bar{x}_{3}=13$ ), the flow of $F_{1}$ is characterized by the well-known tea-cup chaotic attractor (see Figure 2(a)), characterized by LEs $\left\{\lambda_{1} \approx 0.0052, \lambda_{2}=0, \lambda_{3} \approx-0.945\right\}$. As expected from our discussion in section 5 , the trajectories reaching the attractor after a transient sliding (see, e.g., the gray orbit in Figure 2(a)) lose the negative exponent, which is pushed to $-\infty$, while the other 


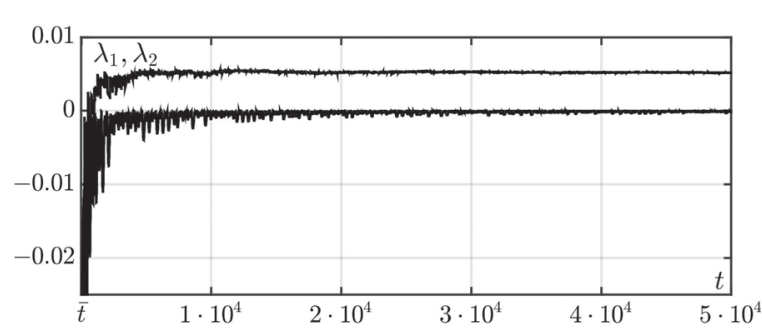

(a)

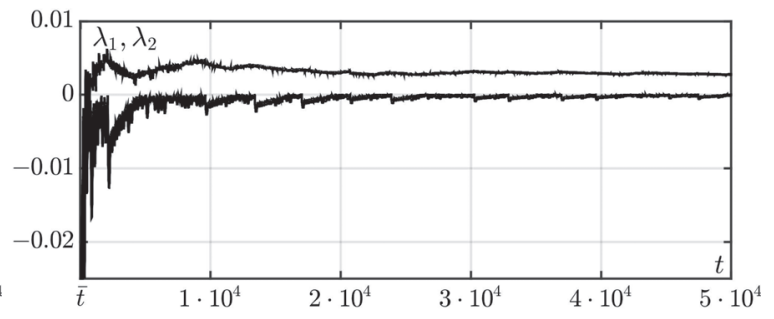

(b)

Figure 3. The rates of convergence of the two nondegenerate LEs. (a) The computation starts at $\mathbf{x}_{0}=$ $(1,0.5,12)^{T}$ and $t_{0}=0$. At $t=\bar{t} \approx 4.4344$ the trajectory first reaches the sliding region, and the corresponding saltation matrix is singular. (b) $\mathbf{x}_{0}=(1,0.5,11)^{T}, t_{0}=0, \bar{t} \approx 4.2000$. Parameter values as in Figure 2.

two exponents converge to the positive and zero exponents of the attractor (see Figure 3(a)). The fact that the most negative exponent of the attractor is the one lost in the trajectories is typical for transient sliding, since the perturbation directions which are not eliminated by the discontinuity tend to align with the leading exponents of the attractor.

Reducing the harvesting threshold $\bar{x}_{3}$, the chaotic attractor eventually grazes the discontinuity manifold $\mathcal{H}$ and then becomes a sliding chaotic attractor (e.g., for $\bar{x}_{3}=12$ in Figure $2(\mathrm{~b}))$. Now, even starting the computation from an initial condition on the attractor, a singular saltation matrix is encountered at the first onset of sliding at time $t=\bar{t}$, setting one exponent to $-\infty$. As expected generically, all the subsequent sliding segments of the reference trajectory imply no further rank loss in the fundamental solution matrix, which remains of rank 2 for $t>\bar{t}$. The two remaining finite LEs are the positive one (giving the chaoticity of the attractor) and the null one (see Figure 3(b)). Note that the largest exponent is smaller than it was before the chaotic attractor hit the discontinuity. This suggests that the discontinuity has a stabilizing effect also in the directions not directly absorbed by the null space of the saltation matrix. This is further supported by reducing the harvesting threshold $\bar{x}_{3}$ even more. Though we don't show this in the plots, a further reduction of $\bar{x}_{3}$ suppresses the chaotic dynamics, which is reduced to a stable limit cycle.

6.2. Vertical hopper. Consider the vertical hopper depicted in Figure 4(a), first proposed by Dankowicz and Piiroinen [20] and later used by Burden, Revzen, and Sastry [13]. The mass $M$ is suspended above the mass $m$ through an actuated linear spring with elastic constant $k$ and nominal length $l_{0}+\sin (w t) a / k$. This could model, for instance, a series elastic linear actuator driven by a periodic control signal. The lower mass is subject to viscous drag with coefficient $b$, and the whole system is subject to gravity. The impacts of the mass $m$ with the ground are assumed perfectly inelastic (i.e., the mass sticks to the ground upon impact). When $m$ is on the ground, the dynamics of the system is given by the equations

$$
F_{1}(\mathbf{x})=\left(\begin{array}{c}
x_{2} \\
\frac{k\left(l_{0}-x_{1}\right)+a \sin \left(w x_{5}\right)-M g}{M} \\
0 \\
0 \\
1
\end{array}\right),
$$




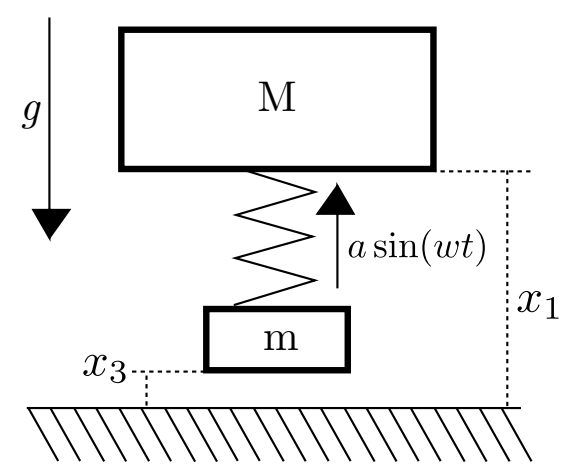

(a)

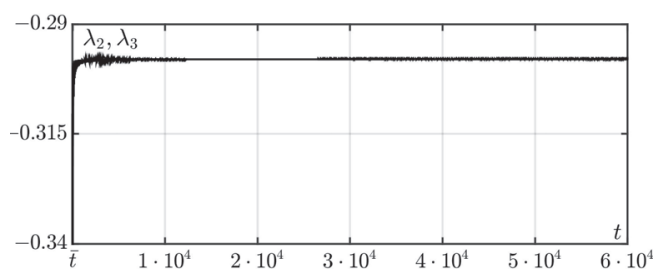

(b)

Figure 4. A sketch of the vertical hopper (a). The rates of convergence of the nondegenerate LEs $\lambda_{2}$ and $\lambda_{3}$ (b). The $\lambda_{1}$ null exponent is not shown. The computation started at $\mathbf{x}_{0}=(1.88,1.96,1,1,0)^{T}$ and $t_{0}=0$. At $t=\bar{t} \approx 2.3201$ a singular transition matrix is met for the first time.

where $x_{1}$ and $x_{3}$ are the distances of the masses $M$ and $m$ from the ground, whereas $x_{2}$ and $x_{4}$ are their velocities. In (6.4) the system has been made time invariant by introducing the phase variable $x_{5}$. The hopper loses contact with the ground when the system reaches $\mathcal{H}_{1}:=$ $\left\{\mathbf{x}: k\left(x_{1}-l_{0}\right)-a \sin \left(w x_{5}\right)-m g=0\right\}$; the $\mathbf{x}$ component of the reset map is $R(\mathbf{x}, 1)=(\mathbf{x}, 2)$.

Once contact with the ground is lost, the system obeys the equations

$$
F_{2}(\mathbf{x})=\left(\begin{array}{c}
x_{2} \\
\frac{k\left(l_{0}+x_{3}-x_{1}\right)+a \sin \left(w x_{5}\right)-M g}{M} \\
x_{4} \\
\frac{k\left(x_{1}-l_{0}-x_{3}\right)-a \sin \left(w x_{5}\right)-b x_{4}-g m}{m} \\
1
\end{array}\right)
$$

until the trajectory reaches $\mathcal{H}_{2}:=\left\{\mathbf{x}: x_{3}=0\right\}$. The corresponding reset map is

$$
R(\mathbf{x}, 2)=\left(\left(\begin{array}{c}
x_{1} \\
x_{2} \\
x_{3} \\
0 \\
x_{5}
\end{array}\right), 1\right) .
$$

The variable $x_{5}$ is reset to 0 by the map when a trajectory reaches the manifold $\mathcal{H}_{3}:=\{\mathbf{x}$ : $\left.H_{3}(\mathbf{x})=x_{5}-2 \pi / \omega=0\right\}$, so we must introduce a third reset map:

$$
R(\mathbf{x}, q)=\left(\left(\begin{array}{c}
x_{1} \\
x_{2} \\
x_{3} \\
x_{4} \\
0
\end{array}\right), q\right) .
$$

The hopper can thus be modelled as a hybrid system with two regions $D_{1}$ and $D_{2}$, and $q$ in (6.6) is 1 or 2 depending on the current region of $\mathbf{x}$. In $D_{1}$ the vector field is (6.4), and the 
discontinuity set is $G_{1}=\mathcal{H}_{1} \cup \mathcal{H}_{3}$; in $D_{2}$ the vector field is (6.5), and the discontinuity set is $G_{2}=\mathcal{H}_{2} \cup \mathcal{H}_{3}$. When the system evolves in $D_{2}$, and $G_{2}$ is reached on $\mathcal{H}_{2}$, it is easy to verify that case (ii) of Theorem 3.1 is satisfied. With reference to the notation of Table 1 , we have ker $R_{2, \mathbf{x}} \subset \operatorname{ker} H_{2, \mathbf{x}}{ }^{6}$ (case (i.c) of Lemma 3.3), and $\mathbf{u} \neq 0$. As a consequence, the corresponding saltation matrix $\mathcal{S}_{2}$ is singular with $\operatorname{dim} \operatorname{ker} \mathcal{S}_{2}=\operatorname{dim} \operatorname{ker} R_{2, \mathrm{x}}+1=2$. All other saltation matrices are invertible.

With parameters $\left(m, M, k, b, l_{0}, a, w, g\right)=(1,2,10,5,2,20,2 \pi, 2)$ the above system exhibits a limit cycle (numerically found in [13]). The LEs are depicted in Figure 4(b). As expected, two exponents are infinitely negative, one is equal to zero, and the remaining two are equal and negative $\left(\lambda_{2}=\lambda_{3} \approx-0.298\right)$.

7. Conclusions. Using the hybrid systems formalism, we have identified the necessary and sufficient conditions for noninvertibility of the fundamental solution matrix in a discontinuous system. We have excluded from our analysis only the cases where the hybrid-flow is not uniquely defined and those where a trajectory reaches a discontinuity set tangentially or where it reaches a corner of the discontinuity set, since in these cases, which are nevertheless nongeneric, we lose the traditional concept of fundamental solution matrix. A comprehensive study of the invertibility conditions for the aforementioned nongeneric cases would require the analysis of an inordinate number of different local configurations, which we leave for future research.

Noninvertibility of the fundamental solution matrix of a reference trajectory implies that the trajectory is infinitely attractive, meaning that some or all perturbations are absorbed in finite time rather than dying out asymptotically. This feature can be used as a means for dimension reduction, as a tool to achieve finite-time convergence, or to stabilize otherwise unstable dynamics. Our result is especially valuable in the light of Theorem 3.1, which states that noninvertibility occurs if and only if one of two simple geometric conditions is verified. This simplifies the task of checking invertibility and of designing systems where a singular fundamental solution matrix is needed. Condition (i) of Theorem 3.1-tangency between a discontinuity manifold and the flow departing from it - is always verified at the onset of sliding in a piecewise smooth discontinuous system. Since these systems appear frequently in mechanics and control theory, we have devoted a section to discussing this special case. Condition (ii) - singularity of an impacting map regulating jumps in state space - is typical of mechanical impacts with no restitution.

A section has been devoted to the relation between flow noninvertibility and LEs. Unlike in smooth systems, where the LEs computed along trajectories converging to the same attractor (almost everywhere) coincide with the attractor's exponents, in hybrid systems there can be open sets of initial conditions for which some of the attractor's exponents (typically the most negative) are "invisible" and replaced with $-\infty$ by the infinite attractiveness of the trajectory. The full history of the discontinuities crossed by a trajectory thus matters, and the resulting LEs are a property of the whole trajectory, rather than of its limit set.

Finally, a numerical procedure which allows the computation of the LEs of a noninvertible flow by resorting to a properly reduced variational system has been presented and applied in two examples.

\footnotetext{
${ }^{6}$ Note that in this particular example ker $R_{2, \mathbf{x}}$ and $\operatorname{ker} H_{2 \mathbf{x}}$ do not depend on the discontinuity point.
} 


\section{REFERENCES}

[1] K. Ainara And H. Suzuki, Theory of hybrid dynamical systems and its applications to biological and medical systems, Phil. Trans. R. Soc. A, 368 (2010), pp. 4893-4914.

[2] M. A. Aizerman and F. R. Gantmakher, On the stability of periodic motions, J. Appl. Math. Mech., 22 (1958), pp. 1065-1078.

[3] I. Belykh, C. Piccardi, and S. Rinaldi, Synchrony in tritrophic food chain metacommunities, J. Biol. Dyn., 3 (2009), pp. 497-514.

[4] A. Ben-Israel and T. N. E. Greville, Generalized Inverses: Theory and Applications, Springer-Verlag, New York, 2003.

[5] G. Benettin, L. Galgani, A. Giorgilli, and J.-M. Strelcyn, Lyapunov characteristic exponents for smooth dynamical systems and for Hamiltonian systems; A method for computing all of them. Part 1: Theory, Meccanica, 15 (1980), pp. 9-20.

[6] G. Benettin, L. Galgani, A. Giorgilli, and J.-M. Strelcyn, Lyapunov characteristic exponents for smooth dynamical systems and for Hamiltonian systems; A method for computing all of them. Part 2: Numerical application, Meccanica, 15 (1980), pp. 21-30.

[7] F. Bizzarri, A. Brambilla, and G. Storti Gajani, Phase noise simulation in analog mixed signal circuits: An application to pulse energy oscillators, IEEE Trans. Circuits Syst. II, Exp. Briefs, 58 (2011), pp. 154-158.

[8] F. Bizzarri, A. Brambilla, and G. Storti Gajani, Periodic small signal analysis of a wide class of type-II phase locked loops through an exhaustive variational model, IEEE Trans. Circuits Syst. I, Reg. Papers, 59 (2012), pp. 2221-2231.

[9] F. Bizzarri, A. Brambilla, and G. Storti Gajani, Steady state computation and noise analysis of analog mixed signal circuits, IEEE Trans. Circuits Syst. I, Reg. Papers, 59 (2012), pp. 541-554.

[10] F. Bizzarri, A. Brambilla, and G. Storti Gajani, Lyapunov exponents computation for hybrid neurons, J. Comput. Neurosci., 35 (2013), pp. 201-212.

[11] M. S. BRAnicky, Topology of hybrid systems, in Proceedings of the 32nd IEEE Conference on Decision and Control, 1993, pp. 2309-2314.

[12] B. Brogliato, Nonsmooth Mechanics: Models, Dynamics and Control, Comm. Control Engrg. Ser., Springer-Verlag, London, 1999.

[13] S. Burden, S. Revzen, And S. S. Sastry, Dimension reduction near periodic orbits of hybrid systems, in Proceedings of the 50th IEEE Conference on Decision and Control, 2011, pp. 6116-6121.

[14] C. Cecchetto And F. Dercole, A new stock market model with adaptive rational equilibrium dynamics, in Proceedings of the IEEE Conference on Complexity in Engineering (COMPENG 2010), Rome, 2010, pp. 129-131.

[15] A. Colombo, Boundary intersection crossing bifurcation in the presence of sliding, Phys. D, 237 (2008), pp. 2900-2912.

[16] A. Colombo, M. di Bernardo, E. Fossas, and M. R. Jeffrey, Teixeira singularities in 3D switched feedback control systems, Systems Control Lett., 59 (2010), pp. 615-622.

[17] A. Colombo, M. di Bernardo, S. J. Hogan, and M. R. Jeffrey, Bifurcations of piecewise smooth flows: Perspectives, methodologies and open problems, Phys. D, 241 (2012), pp. 1845-1860.

[18] A. Colombo And M. R. JefFrey, Nondeterministic chaos, and the two-fold singularity in piecewise smooth flows, SIAM J. Appl. Dyn. Syst., 10 (2011), pp. 423-451.

[19] A. Colombo and S. Rinaldi, Chaos in two-party democracies, Int. J. Bifurcat. Chaos, 18 (2008), pp. 2133-2140.

[20] H. Dankowicz And P. T. Pirroinen, Exploiting discontinuities for stabilization of recurrent motions, Dyn. Syst., 17 (2002), pp. 317-342.

[21] S. L.T. DE SouzA AND I. L. CALDAs, Calculation of Lyapunov exponents in systems with impacts, Chaos Solitons Fractals, 19 (2004), pp. 569-579.

[22] F. Dercole, R. Ferrière, And S. Rinaldi, Ecological bistability and evolutionary reversals under asymmetrical competition, Evolution, 56 (2002), pp. 1081-1090.

[23] F. Dercole, R. Ferrière, A. Gragnani, and S. Rinaldi, Coevolution of slow-fast populations: Evolutionary sliding, evolutionary pseudo-equilibria and complex Red Queen dynamics, Proc. R. Soc. Lond. Ser. B, 273 (2006), pp. 983-990. 
[24] F. Dercole, A. Gragnani, Yu. A. Kuznetsov, and S. Rinaldi, Numerical sliding bifurcation analysis: An application to a relay control system, IEEE Trans. Circuits Syst. I, Fundam. Theory Appl., 50 (2003), pp. 1058-1063.

[25] F. Dercole, A. Gragnani, And S. Rinaldi, Bifurcation analysis of piecewise smooth ecological models, Theor. Popul. Biol., 72 (2007), pp. 197-213.

[26] M. di Bernardo, C. J. Budd, And A. R. Champneys, Corner collision implies border-collision bifurcation, Phys. D, 154 (2001), pp. 171-194.

[27] M. Di Bernardo, C. J. Budd, ANd A. R. Champneys, Grazing bifurcations in n-dimensional piecewisesmooth dynamical systems, Phys. D, 160 (2001), pp. 222-254.

[28] M. di Bernardo, C. J. Budd, A. R. Champneys, and P. Kowalczyk, Piecewise-smooth Dynamical Systems. Theory and Applications, Springer-Verlag, London, 2008.

[29] M. di Bernardo, A. Colombo, And E. Fossas, Two-fold singularity in nonsmooth electrical systems, in Proceedings of the 2011 IEEE International Symposium on Circuits and Systems, 2011, pp. 27132716.

[30] L. Dieci And L. Lopez, Fundamental matrix solutions of piecewise smooth differential systems, Math. Comput. Simulation, 81 (2011), pp. 932-953.

[31] L. Dieci, R. D. Russell, and E. S. Van Vleck, On the computation of Lyapunov exponents for continuous dynamical systems, SIAM J. Numer. Anal., 34 (1997), pp. 402-423.

[32] A. F. Filippov, Differential equations with discontinuous right-hand side, Mat. Sb. (N.S.), 51 (1960), pp. $99-128$.

[33] A. F. Filippov, Differential Equations with Discontinuous Righthand Sides, Kluwer Academic Publishers, Dordrecht, The Netherlands, 1988.

[34] W. J. F. Govaerts, Numerical Methods for Bifurcations of Dynamical Equilibria, SIAM, Philadelphia, 2000.

[35] A. Hastings and T. Powell, Chaos in a three-species food chain, Ecology, 72 (1991), pp. 896-903.

[36] W. P. M. H. Heemels, B. De Scutter, And A. Bemporad, Equivalence of hybrid dynamical models, Automatica J. IFAC, 37 (2001), pp. 1085-1091.

[37] N. Hinrichs, M. Oestreich, And K. Popp, Dynamics of oscillators with impact and friction, Chaos Solitons Fractals, 8 (1997), pp. 535-558.

[38] D. Hristu-Varsakelis, W. S. Levine, R. Alur, K.-E. Arzen, J. Baillieul, and T. A. Henzinger, Handbook of Networked and Embedded Control Systems (Control Engineering), Birkhäuser, Basel, 2005.

[39] A. P. Ivanov, The stability of periodic solutions of discontinuous systems that intersect several surfaces of discontinuity, J. Appl. Math. Mech., 62 (1998), pp. 677-685.

[40] E. M. Izhikevich, Dynamical Systems in Neuroscience: The Geometry of Excitability and Bursting, MIT Press, Cambridge, MA, 2007.

[41] M. R. Jeffrey And A. Colombo, The two-fold singularity of discontinuous vector fields, SIAM J. Appl. Dyn. Syst., 8 (2009), pp. 624-640.

[42] L. Levaggi and S. Villa, On the regularization of sliding modes, SIAM J. Optim., 18 (2007), pp. 878894.

[43] V. H. Linh And V. Mehrmann, Spectral analysis for linear differential-algebraic equations, Discrete Contin. Dyn. Syst., Supp. (2011), pp. 991-1000.

[44] V. H. Linh, V. Mehrmann, And E. S. VAn Vleck, QR methods and error analysis for computing Lyapunov and Sacker-Sell spectral intervals for linear differential-algebraic equations, Adv. Comput. Math., 35 (2011), pp. 281-322.

[45] S. Liu And L.-Q. Chen, Second-order terminal sliding mode control for networks synchronization, Nonlinear Dynam., 79 (2015), pp. 205-213.

[46] P. C. MüLLER, Calculation of Lyapunov exponents for dynamic systems with discontinuities, Chaos Solitons Fractals, 5 (1995), pp. 1671-1681.

[47] A. B. Nordmark, Non-periodic motion caused by grazing incidence in an impact oscillator, J. Sound Vibration, 145 (1991), pp. 279-297.

[48] T. S. Parker And L. O. ChuA, Practical Numerical Algorithms for Chaotic Systems, Springer-Verlag, New York, 1989.

[49] S. N. Simic, K. H. Johansson, J. Lygeros, And S. Sastry, Structural stability of hybrid systems, in Proceedings of the 2001 European Control Conference, IEEE, Piscataway, NJ, 2001, pp. 3858-3863. 
[50] S. N. Simić, K. H. Johansson, J. Lygeros, and S. Sastry, Towards a geometric theory of hybrid systems, Dyn. Contin. Discrete Impuls. Syst. Ser. B Appl. Algorithms, 12 (2005), pp. 649-687.

[51] V. I. UtKIn, Variable structure systems with sliding modes, IEEE Trans. Automat. Control, 22 (1977), pp. $212-222$.

[52] A. Wolf, J. B. Swift, H. L. Swinney, And J. A. Vastano, Determining Lyapunov exponents from a time series, Phys. D, 16 (1985), pp. 285-317.

[53] J. Zhang, K. H. Johansson, J. Lygeros, and S. Sastry, Zeno hybrid systems, Internat. J. Robust Nonlinear Control, 11 (2001), pp. 435-451.

[54] D. Zhou, Y. Sun, A. V. Rangan, And D. CaI, Spectrum of Lyapunov exponents of non-smooth dynamical systems of integrate-and-fire type, J. Comput. Neurosci., 28 (2010), pp. 229-245. 